International Electronic Journal of Algebra

Volume 26 (2019) 145-190

DOI: $10.24330 /$ ieja. 587041

\title{
SEMIGROUPS GENERATED BY PARTITIONS
}

\author{
O. Dovgoshey
}

Received: 18 January 2019; Accepted: 19 May 2019

Communicated by Abdullah Harmancı

\begin{abstract}
Let $X$ be a nonempty set and $X^{2}$ be the Cartesian square of $X$. Some semigroups of binary relations generated by partitions of $X^{2}$ are studied. In particular, the algebraic structure of semigroups generated by the finest partition of $X^{2}$ and, respectively, by the finest symmetric partition of $X^{2}$ are described.
\end{abstract}

Mathematics Subject Classification (2010): 20M05

Keywords: Partition of a set, semigroup of binary relations, band of semigroups

\section{Introduction}

Let $X$ be a set. A binary relation on $X$ is a subset of the Cartesian square

$$
X^{2}=X \times X=\{\langle x, y\rangle: x, y \in X\} .
$$

The composition of binary relations $\psi$ and $\gamma$ on $X$ is a binary relation $\psi \circ \gamma \subseteq X \times X$ for which $\langle x, y\rangle \in \psi \circ \gamma$ holds if and only if there is $z \in X$ such that $\langle x, z\rangle \in \psi$ and $\langle z, y\rangle \in \gamma$. It is well-known that $\circ$ is an associative operation on the set of binary relations on $X$.

Recall that a semigroup is a pair $(\mathcal{S}, *)$ consisting of a nonempty set $\mathcal{S}$ and an associative operation $*: \mathcal{S} \times \mathcal{S} \rightarrow \mathcal{S}$ which is called the multiplication on $\mathcal{S}$. As usual, we use the symbol $x * y$ instead of $*\langle x, y\rangle$ to indicate the result of applying $*$ to $\langle x, y\rangle$. A semigroup $\mathcal{S}=(\mathcal{S}, *)$ is a monoid if there is $e \in \mathcal{S}$ such that

$$
e * s=s * e=s
$$

for every $s \in \mathcal{S}$. In this case we say that $e$ is the identity element of the semigroup $(\mathcal{S}, *)$. A zero of a semigroup $(\mathcal{S}, *)$ is an element $\theta \in \mathcal{S}$ for which

$$
\theta * s=s * \theta=\theta
$$

holds for every $s \in \mathcal{S}$. A set $A \subseteq \mathcal{S}$ is a set of generators of $(\mathcal{S}, *)$ if, for every $s \in \mathcal{S}$, there is a finite sequence $s_{1}, \ldots, s_{k}$ of elements of $A$ such that

$$
s=s_{1} * \ldots * s_{k} .
$$


A nonempty subset $B$ of $\mathcal{S}$ is a subsemigroup of $(\mathcal{S}, *)$ if $x * y \in B$ holds for all $x$, $y \in B$.

We denote by $\mathcal{B}_{X}=\left(\mathcal{B}_{X}, \circ\right)$ the semigroup of all binary relations defined on a set $X$ such that the composition $\circ$ of relations is the multiplication on $\mathcal{B}_{X}$. It is well-known that every semigroup $(\mathcal{H}, *)$ is isomorphic to a subsemigroup of $\mathcal{B}_{X}$ for a suitable $X$. The properties of $\mathcal{B}_{X}$ have been investigated by many mathematicians $[4,5,8-10,21,23,26,28,29,31-34,36,38,39]$. In particular, the minimal generating sets for $\mathcal{B}_{X}$ were considered in [7] and [24]. The so-called complete semigroups of binary relations are investigated by Yasha Diasamidze, Shota Makharadze et al. (see, for example, $[1,3,8,11-16]$ ).

Following [27] we say that a set $B$ of binary relations on a set $X$ is transitive if for every $\langle x, y\rangle \in X \times X$ there is $R \in B$ such that $\langle x, y\rangle \in R$. A homomorphism $\Phi: \mathcal{S} \rightarrow \mathcal{B}_{X}$ of $(\mathcal{S}, *)$ is called transitive if $\Phi(\mathcal{S})$ is a transitive set of relations. A faithful representation of a semigroup $(\mathcal{S}, *)$ by binary relations is a monomorphism $\mathcal{S} \rightarrow \mathcal{B}_{X}$

Solving a longstanding problem formulated in [35] Ralph McKenzie and Boris Schein prove that all semigroups have faithful transitive representations [27]. Like every outstanding result, the McKenzie-Shein theorem raises a series of related questions. According to this theorem, for every semigroup $(\mathcal{H}, *)$ there are a monomorphism $\Phi: \mathcal{S} \rightarrow \mathcal{B}_{X}$ and a set $A$ of generators of $\mathcal{S}$ such that $\Phi(A)$ is a cover of $X^{2}$. What can be said about the properties of this cover? In particular, under what conditions is $\Phi(A)$ a partition of $X^{2}$ ?

Definition 1.1. A monomorphism $\Phi: \mathcal{S} \rightarrow \mathcal{B}_{X}$ is d-transitive (disjoint-transitive) if there is a set $A$ of generators of $(\mathcal{S}, *)$ such that $\{\Phi(a): a \in A\}$ is a partition of the set $X^{2}$ and, if $(\mathcal{S}, *)$ contains a zero element $\theta$, the equality $\Phi(\theta)=\varnothing$ holds.

It is clear that every $d$-transitive monomorphism $\mathcal{S} \rightarrow \mathcal{B}_{X}$ is a faithful and transitive representation of $(\mathcal{S}, *)$.

The following problem seems to be interesting and this is the main object of research in the paper.

Problem 1.2. Describe the algebraic structure of semigroups $(\mathcal{H}, *)$ admitting $d$-transitive monomorphisms $\mathcal{H} \rightarrow \mathcal{B}_{X}$.

The paper is organized as follows.

In Section 2 we consider some basic partitions of Cartesian squares of sets and describe properties of these partitions.

The main results of the paper are formulated and proved in Section 3 and Section 4. Theorem 3.2, Proposition 3.11 and Theorem 4.6 give us a "purely algebraic" description of some classes of semigroups $\mathcal{H}$ admitting $d$-transitive monomorphisms $\mathcal{H} \rightarrow \mathcal{B}_{X}$ for suitable $X$. 
Examples of semigroups $\mathcal{H}$ which have no $d$-transitive monomorphisms $\mathcal{H} \rightarrow \mathcal{B}_{X}$ are given in Proposition 3.9 and Proposition 4.14.

\section{Partitions of Cartesian square}

Let $X$ be a nonempty set and $P=\left\{X_{j}: j \in J\right\}$ be a set of nonempty subsets of $X$. The set $P$ is a partition of $X$ if we have

$$
\bigcup_{j \in J} X_{j}=X \quad \text { and } \quad X_{j_{1}} \cap X_{j_{2}}=\varnothing
$$

for all distinct $j_{1}, j_{2} \in J$. In what follows we will say that the sets $X_{j}, j \in J$ are the blocks of $P$.

We say that partitions $P=\left\{X_{j}: j \in J\right\}$ and $Q=\left\{X_{i}: i \in I\right\}$ of a set $X$ are equal if and only if there is a bijective mapping $f: J \rightarrow I$ such that $X_{j}=X_{f(j)}$ holds for every $j \in J$.

Example 2.1. Let $X$ and $Y$ be a nonempty sets. If a mapping $\Psi: X \rightarrow Y$ is surjective, then the set

$$
P_{\Psi^{-1}}:=\left\{\Psi^{-1}(y): y \in Y\right\}
$$

is a partition of $X$ with blocks $\Psi^{-1}(y), y \in Y$. Conversely, if $P=\left\{X_{j}: j \in J\right\}$ is a partition of $X$, then the mapping $F: X \rightarrow J$ defined by

$$
(F(x)=j) \Leftrightarrow\left(x \in X_{j}\right)
$$

is surjective and the equality $P=P_{F^{-1}}$ holds.

Let $X$ be a set. A binary relation $R \subseteq X \times X$ is an equivalence relation on $X$ if the following conditions hold for all $x, y, z \in X$ :

(i) $\langle x, x\rangle \in R$, the reflexive law;

(ii) $(\langle x, y\rangle \in R) \Leftrightarrow(\langle y, x\rangle \in R)$, the symmetric law;

(iii) $((\langle x, y\rangle \in R)$ and $(\langle y, z\rangle \in R)) \Rightarrow(\langle x, z\rangle \in R)$, the transitive law.

If $R$ is an equivalence relation on $X$, then an equivalence class is defined as a subset $[a]_{R}$ of $X$ having the form

$$
[a]_{R}=\{x \in X:\langle x, a\rangle \in R\}, a \in X .
$$

There exists the well-known, one-to-one correspondence between the equivalence relations and the partitions (see, for example, [25, Chapter II, § 5] or [19, Proposition 1.4.6]).

Proposition 2.2. Let $X$ be a nonempty set. If $P=\left\{X_{j}: j \in J\right\}$ is a partition of $X$ and $R_{P}$ is a binary relation on $X$ such that, for every $\langle x, y\rangle \in X \times X$,

$$
\left(\langle x, y\rangle \in R_{P}\right) \Leftrightarrow\left(\exists j \in J\left(x \in X_{j} \text { and } y \in X_{j}\right)\right),
$$


then $R_{P}$ is an equivalence relation on $X$ with equivalence classes $X_{j}$. Conversely, if $R$ is an equivalence relation on $X$, then the set $P_{R}$ of all distinct equivalence classes $[a]_{R}$ is a partition of $X$ with blocks $[a]_{R}$.

Remark 2.3. If $X=\varnothing$, then we have $X \times X=\varnothing$, so that $\varnothing$ can be considered as a unique equivalence relation on $\varnothing$. It should be noted that there is no partition of $\varnothing$ because every block of each partition is nonempty by definition.

In the following, we systematically use the notation $R_{P}$ for the equivalence relation corresponding to partition $P$ and the notation $P_{R}$ for the partition corresponding to equivalence relation $R$. In particular, for every nonempty set $X$, Proposition 2.2 implies the equality

$$
P=P_{R_{P}}
$$

if $P$ is a given partition of $X$ and, respectively, the equality

$$
R=R_{P_{R}}
$$

if $R$ is a given equivalence relation on $X$.

The trivial examples of equivalence relations on $X$ are the Cartesian square $X^{2}$ and the diagonal $\Delta_{X}$ of $X$,

$$
\Delta_{X}:=\{\langle x, x\rangle: x \in X\}
$$

If $X$ is nonempty, then we have

$$
P_{\Delta_{X}}=\{\{x\}: x \in X\} .
$$

Moreover, for a partition $P=\left\{X_{j}: j \in J\right\}$ of $X$, the equality $P=P_{X^{2}}$ holds if and only if $|J|=1$.

In the following proposition starting from a partition $P$ of a set $X$ we define a partition $P \otimes P$ of the Cartesian square $X^{2}$.

Proposition 2.4. Let $X$ be a nonempty set and $P=\left\{X_{j}: j \in J\right\}$ be a partition of $X$. Write

$$
P \otimes P:=\left\{X_{j_{1}} \times X_{j_{2}}:\left\langle j_{1}, j_{2}\right\rangle \in J^{2}\right\}
$$

where $X_{j_{1}} \times X_{j_{2}}$ is the Cartesian product of $X_{j_{1}}$ and $X_{j_{2}}$, and $J^{2}$ is the Cartesian square of $J$. Then $P \otimes P$ is a partition of the Cartesian square $X^{2}$ with blocks $X_{j_{1}} \times X_{j_{2}},\left\langle j_{1}, j_{2}\right\rangle \in J^{2}$.

The proof is simple and we omit it here.

Example 2.5. Let $X$ be a nonempty set and let

$$
P_{\Delta_{X}}=\{\{x\}: x \in X\}
$$


be a partition of $X$ corresponding to the diagonal $\Delta_{X}$ on $X$. Then $P_{\Delta_{X}} \otimes P_{\Delta_{X}}$ is a partition of $X^{2}$ corresponding to the diagonal on $X^{2}$,

$$
P_{\Delta_{X}} \otimes P_{\Delta_{X}}=P_{\Delta_{X^{2}}}=\left\{\{\langle x, y\rangle\}:\langle x, y\rangle \in X^{2}\right\} .
$$

Proposition 2.6. Let $X$ be a nonempty set. If $R$ is an equivalence relation on $X$ and $P_{R}=\left\{X_{j}: j \in J\right\}$ is the corresponding partition of $X$, then the equality

$$
R=\bigcup_{j \in J} X_{j}^{2}
$$

holds.

Proof. Let $R$ be an equivalence relation on $X$ and $P_{R}=\left\{X_{j}: j \in J\right\}$. The set $\bigcup_{j \in J} X_{j}^{2}$ is a subset of $X^{2}$ and, consequently, it is a binary relation on $X$. By Proposition 2.2, for every $\left\langle x_{1}, y_{1}\right\rangle \in R$ there is $j_{1} \in J$ such that $x_{1} \in X_{j_{1}}$ and $y_{1} \in X_{j_{1}}$, i.e.,

$$
\left\langle x_{1}, y_{1}\right\rangle \in X_{j_{1}}^{2} \subseteq \bigcup_{j \in J} X_{j}^{2} .
$$

It implies the inclusion

$$
R \subseteq \bigcup_{j \in J} X_{j}^{2}
$$

Now let $\left\langle x_{0}, y_{0}\right\rangle$ be an arbitrary point of $\bigcup_{j \in J} X_{j}^{2}$. Then there is $j_{0} \in J$ such that $\left\langle x_{0}, y_{0}\right\rangle \in X_{j_{0}}^{2}$, which means $x_{0} \in X_{j_{0}}$ and $y_{0} \in X_{j_{0}}$. Since $\left\{X_{j}: j \in J\right\}$ is the partition corresponding to $R$, we have $\left\langle x_{0}, y_{0}\right\rangle \in R$ by Proposition 2.2. Consequently, the inclusion

$$
\bigcup_{j \in J} X_{j}^{2} \subseteq R
$$

holds. The last inclusion and (2.7) imply (2.6).

Example 2.7. Let $X$ be a nonempty set. Then the equality

$$
\Delta_{X}=\{\{x\} \times\{x\}: x \in X\}
$$

holds.

For every partition $P=\left\{X_{j}: j \in J\right\}$ of a nonempty set $X$ we define a partition $P \otimes P^{1}$ of $X^{2}$ as

$$
P \otimes P^{1}:=\left\{R_{P}\right\} \cup\left\{X_{j_{1}} \times X_{j_{2}}:\left\langle j_{1}, j_{2}\right\rangle \in \nabla_{J}\right\},
$$

where $\Delta_{J}$ is the diagonal of $J$ and $\nabla_{J}:=J^{2} \backslash \Delta_{J}$. We will also consider partitions $P \otimes P_{S}$ and $P \otimes P_{S}^{1}$ defined by the rules:

(i) A subset $B$ of $X^{2}$ is a block of $P \otimes P_{S}$ if and only if there are $j_{1}, j_{2} \in J$ such that

$$
B=\left(X_{j_{1}} \times X_{j_{2}}\right) \cup\left(X_{j_{2}} \times X_{j_{1}}\right)
$$


(ii) A subset $B$ of $X^{2}$ is a block of $P \otimes P_{S}^{1}$ if and only if either $B=R_{P}$ or there are distinct $j_{1}, j_{2} \in J$ such that (2.9) holds.

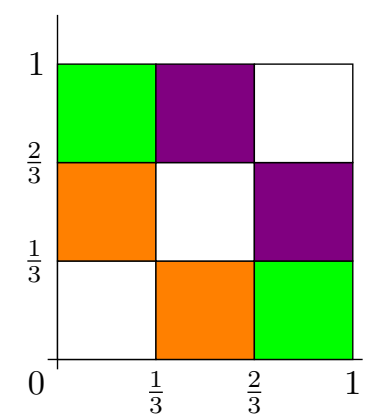

Figure 1. The partition $P \otimes P_{S}^{1}$ corresponding to trichotomy $P=$ $\left\{X_{0}, X_{1}, X_{2}\right\}$. Here $R_{P}$ is white, $R_{1}$ is orange, $R_{2}$ is green and $R_{3}$ is violet.

Example 2.8. Let $\mathbb{R}$ be the field of real numbers and let

$$
\begin{array}{rlrl}
X & :=\{x \in \mathbb{R}: 0 \leqslant x \leqslant 1\}, & X_{0}:=\left\{x \in \mathbb{R}: 0 \leqslant x \leqslant \frac{1}{3}\right\}, \\
X_{1}:=\left\{x \in \mathbb{R}: \frac{1}{3}<x<\frac{2}{3}\right\}, & X_{2}:=\left\{x \in \mathbb{R}: \frac{2}{3} \leqslant x \leqslant 1\right\} .
\end{array}
$$

Then the trichotomy $P=\left\{X_{0}, X_{1}, X_{2}\right\}$ is a partition of $X$. Write

$$
\begin{array}{ll}
R_{1}:=\left(X_{0} \times X_{1}\right) \cup\left(X_{1} \times X_{0}\right), & R_{2}:=\left(X_{0} \times X_{2}\right) \cup\left(X_{2} \times X_{0}\right), \\
R_{3}:=\left(X_{1} \times X_{2}\right) \cup\left(X_{2} \times X_{1}\right), & R_{P}:=\bigcup_{j=0}^{2} X_{j}^{2} .
\end{array}
$$

Then $P \otimes P_{S}^{1}=\left\{R_{P}, R_{1}, R_{2}, R_{3}\right\}$ holds (see Figure 1 ).

The partitions $P \otimes P, P \otimes P^{1}, P \otimes P_{S}$ and $P \otimes P_{S}^{1}$ can be characterized as the smallest elements of corresponding subsets of the partially ordered set of all partitions of $X^{2}$.

Definition 2.9. Let $X$ be a nonempty set and let $P_{1}$ and $P_{2}$ be partitions of $X$. The partition $P_{1}$ is finer than the partition $P_{2}$ if the inclusion

$$
[x]_{R_{P_{1}}} \subseteq[x]_{R_{P_{2}}}
$$

holds for every $x \in X$, where $R_{P_{1}}$ and $R_{P_{2}}$ are equivalence relations corresponding to $P_{1}$ and $P_{2}$ respectively.

If $P_{1}$ is finer than $P_{2}$, then we write $P_{1} \leqslant{ }_{X} P_{2}$ and say that $P_{1}$ is a refinement of $P_{2}$. 
Remark 2.10. Using Proposition 2.6, we see that if $P_{1}$ and $P_{2}$ are partitions of $X$, then $P_{1}$ is a refinement of $P_{2}$ if and only if the inclusion $R_{P_{1}} \subseteq R_{P_{2}}$ holds.

Example 2.11. Let $X$ be a nonempty set and let $P$ be a partition of $X$. Then

$$
P \otimes P \leqslant X^{2} P \otimes P^{1} \leqslant X^{2} P \otimes P_{S}^{1}
$$

and

$$
P \otimes P \leqslant X^{2} P \otimes P_{S} \leqslant X^{2} P \otimes P_{S}^{1}
$$

hold.

Recall that a reflexive and transitive binary relation $\preccurlyeq$ on a set $Y$ is a partial order on $Y$ if the following antisymmetric law

$$
(\langle x, y\rangle \in \preccurlyeq \text { and }\langle y, x\rangle \in \preccurlyeq) \Rightarrow(x=y)
$$

holds for all $x, y \in Y$.

In what follows, for partial order $\preccurlyeq$, we write $x \preccurlyeq y$ instead of $\langle x, y\rangle \in \preccurlyeq$.

The following proposition is well-known (see, for example, [25, Example 4, § 9 Chapter 2]) and directly follows from Remark 2.10.

Proposition 2.12. Let $X$ be a nonempty set and let $\Pi(X)$ be the set of all partitions of $X$. Then the binary relation "to be finer than" is a partial order on $\Pi(X)$.

Remark 2.13. The partially ordered set $\left(\boldsymbol{\Pi}(X), \leqslant_{X}\right)$ of all partitions of $X$ is a complete lattice (see Section 13.3 of [2]). The theory of lattices of partitions of a given set was developed by Oystein Ore in [30]. In particular, Ore has characterized the lattices which are isomorphic to lattice $\left(\boldsymbol{\Pi}(X), \leqslant_{X}\right)$ for some set $X$. It was shown by Philip M. Whitman [37] that every lattice is isomorphic to a sublattice of $(\boldsymbol{\Pi}(X), \leqslant X)$ for a suitable $X$.

The following lemma is straightforward.

Lemma 2.14. Let $X$ be a nonempty set and let $P_{1}, P_{2} \in \Pi(X)$. Then the following statements are equivalent.

(i) $P_{1} \leqslant X P_{2}$.

(ii) If $\Psi_{1}: X \rightarrow Y_{1}$ and $\Psi_{2}: X \rightarrow Y_{2}$ are surjective mappings such that

$$
P_{1}=P_{\Psi_{1}^{-1}} \quad \text { and } \quad P_{2}=P_{\Psi_{2}^{-1}}
$$

(see (2.1)), then there is a surjective mapping $\Phi: Y_{1} \rightarrow Y_{2}$ for which

$$
\Psi_{2}(x)=\Phi\left(\Psi_{1}(x)\right)
$$

holds for every $x \in X$.

(iii) Each block of $P_{2}$ is a union of some blocks of $P_{1}$. 
Definition 2.15. Let $X$ be a nonempty set, let $R$ be an equivalence relation on $X$ and let $\Phi$ be a mapping with domain $X^{2}$. The mapping $\Phi$ is $R$-coherent if the implication

$$
\left(\left\langle x_{1}, x_{3}\right\rangle \in R \text { and }\left\langle x_{2}, x_{4}\right\rangle \in R\right) \Rightarrow\left(\Phi\left(x_{1}, x_{2}\right)=\Phi\left(x_{3}, x_{4}\right)\right)
$$

is valid for all $x_{1}, x_{2}, x_{3}, x_{4} \in X$.

For a given set $X$ and a given equivalence relation $R$ on $X$, we denote by $\operatorname{Coh}(R)$ the class of all surjective, $R$-coherent mappings with domain $X^{2}$.

Now we recall a notion of the smallest element of a subset of a partially ordered set. Let $(Y, \preccurlyeq)$ be a partially ordered set and let $A \subseteq Y$. An element $a^{*} \in A$ is called to be the smallest element of $A$ if

$$
a^{*} \preccurlyeq a
$$

holds for every $a \in A$. It is easy to see that the smallest element of $A$ is unique if it exists.

The following simple theorem characterizes $P \otimes P$ as the smallest element of the set of all partitions of $X^{2}$ generated by mappings belonging to $\operatorname{Coh}\left(R_{P}\right)$.

Theorem 2.16. Let $X$ be a nonempty set and let $Q=\left\{X_{j}: j \in J\right\}$ be a partition of $X$. Then the inequality

$$
Q \otimes Q \leqslant X^{2} P_{F^{-1}}
$$

holds for every $F \in \operatorname{Coh}\left(R_{Q}\right)$ and, moreover, there is $F_{0} \in \operatorname{Coh}\left(R_{Q}\right)$ such that the equality

holds.

$$
Q \otimes Q=P_{F_{0}^{-1}}
$$

Proof. Let $F \in \operatorname{Coh}\left(R_{Q}\right)$. Inequality (2.11) holds if and only if we have

$$
X_{j_{1}} \times X_{j_{2}} \subseteq F^{-1}\left(F\left(x_{1}, x_{2}\right)\right)
$$

for every $\left\langle x_{1}, x_{2}\right\rangle \in X \times X$, where $X_{j_{1}} \times X_{j_{2}}$ is a block of $Q \otimes Q$ such that $\left\langle x_{1}, x_{2}\right\rangle \in$ $X_{j_{1}} \times X_{j_{2}}$. Suppose $\left\langle x_{3}, x_{4}\right\rangle$ is an arbitrary point of $X_{j_{1}} \times X_{j_{2}}$. Then we have

$$
x_{1}, x_{3} \in X_{j_{1}} \quad \text { and } \quad x_{2}, x_{4} \in X_{j_{2}}
$$

that implies $\left\langle x_{1}, x_{3}\right\rangle \in R_{Q}$ and $\left\langle x_{2}, x_{4}\right\rangle \in R_{P}$. Since $F$ is $R_{Q}$-coherent, the equality $F\left(x_{1}, x_{2}\right)=F\left(x_{3}, x_{4}\right)$ holds. Thus, we have

$$
\left\langle x_{3}, x_{4}\right\rangle \in F^{-1}\left(F\left(x_{1}, x_{2}\right)\right) .
$$

Inclusion (2.12) follows.

Let us consider $F_{0}: X^{2} \rightarrow J^{2}$ such that

$$
\left(F_{0}(x, y)=\left\langle j_{1}, j_{2}\right\rangle\right) \Leftrightarrow\left(x \in X_{j_{1}} \text { and } y \in X_{j_{2}}\right)
$$


is valid for every $\langle x, y\rangle \in X^{2}$. Let $x_{1}, x_{2}, x_{3}, x_{4}$ belong to $X$. If $\left\langle x_{1}, x_{3}\right\rangle \in R_{Q}$ and $\left\langle x_{2}, x_{4}\right\rangle \in R_{Q}$ then, by Proposition 2.6, there are $j_{1} \in J$ and $j_{2} \in J$ such that

$$
x_{1}, x_{3} \in X_{j_{1}} \text { and } x_{2}, x_{4} \in X_{j_{2}} .
$$

Using these membership relations and (2.13) we obtain

$$
F_{0}\left(x_{1}, x_{2}\right)=\left\langle j_{1}, j_{2}\right\rangle=F_{0}\left(x_{3}, x_{4}\right) .
$$

Hence, $F_{0} \in \operatorname{Coh}\left(R_{Q}\right)$ holds. It follows from (2.13) that

$$
Q \otimes Q=\left\{F_{0}^{-1}\left(\left\langle j_{1}, j_{2}\right\rangle\right):\left\langle j_{1}, j_{2}\right\rangle \in J^{2}\right\} .
$$

Example 2.17. Let $X$ be a nonempty set and let

$$
P=P_{\Delta_{X}}=\{\{x\}: x \in X\}
$$

be a partition of $X$ corresponding to the diagonal $\Delta_{X}$ on $X$. Then $P \otimes P$ is a partition corresponding to the diagonal $\Delta_{X^{2}}$ on $X^{2}$ (see Example 2.5). Hence, the inequality

$$
P \otimes P \leqslant X^{2} Q
$$

holds for every partition $Q \in \Pi\left(X^{2}\right)$ and, consequently, every mapping with domain $X^{2}$ is $\Delta_{X}$-coherent.

Recall that for every nonempty $A \subseteq \mathcal{B}_{X}$ we write $\mathcal{S}_{A}$ for the subsemigroup of $\left(\mathcal{B}_{X}, \circ\right)$ having $A$ as a set of generators.

Proposition 2.18. If a semigroup $(\mathcal{H}, *)$ admits a d-transitive monomorphism $\mathcal{H} \rightarrow \mathcal{B}_{X}$, then there are an equivalence relation $R$ on $X$ and a mapping $\Phi \in \operatorname{Coh}(R)$ such that $(\mathcal{H}, *)$ and $\mathcal{S}_{A}$ are isomorphic with $A=P_{\Phi^{-1}}$.

Proof. Let $F: \mathcal{H} \rightarrow \mathcal{B}_{X}$ be a $d$-transitive monomorphism of $(\mathcal{H}, *)$ and let $A$ be a set of generators of $\mathcal{H}$ such that

$$
Q:=\{F(a): a \in A\}
$$

is a partition of $X^{2}$. As in Example 2.17, we see that the inequality

$$
P \otimes P \leqslant X^{2} Q
$$

holds with $P=\{\{x\}: x \in X\}$. Now the proposition follows from Theorem 2.16.

Let $X$ be a nonempty set and let $R$ be an equivalence relation on $X$. Let us denote by $\operatorname{Coh}^{1}(R)$ a subclass of mappings of the class $\operatorname{Coh}(R)$ such that $\Phi \in \operatorname{Coh}^{1}(R)$ if and only if $\Phi \in \operatorname{Coh}(R)$ and

$$
\Phi(x, x)=\Phi(y, y) \text { holds for all } x, y \in X .
$$


Analogously to Theorem 2.16 we can characterize $P \otimes P^{1}$ as the smallest element of the set of all partitions of $X^{2}$ generated by mappings belonging to $\operatorname{Coh}^{1}\left(R_{P}\right)$.

Theorem 2.19. Let $X$ be a nonempty set and let $Q=\left\{X_{j}: j \in J\right\}$ be a partition of $X$. Then the inequality

$$
Q \otimes Q^{1} \leqslant X^{2} P_{F^{-1}}
$$

holds for every $F \in \operatorname{Coh}^{1}\left(R_{Q}\right)$ and, moreover, there is $F_{1} \in \operatorname{Coh}^{1}\left(R_{Q}\right)$ such that the equality

$$
Q \otimes Q^{1}=P_{F_{1}^{-1}}
$$

holds.

Proof. Arguing as in the proof of inequality (2.11), it is easy to make sure that (2.15) is true for every $F \in \operatorname{Coh}^{1}\left(R_{Q}\right)$.

Write

$$
\nabla_{J}:=J^{2} \backslash \Delta_{J} \quad \text { and } \quad J^{2,1}:=\nabla_{J} \cup\left\{\Delta_{J}\right\}
$$

i.e., the diagonal $\Delta_{J}$ is deleted from the Cartesian square $J^{2}$ and the single-point set $\left\{\Delta_{J}\right\}$ is added to the set-theoretic difference of $J^{2}$ and $\Delta_{J}$. Let us consider $\Psi: J^{2} \rightarrow J^{2,1}$ such that

$$
\Psi\left(\left\langle j_{1}, j_{2}\right\rangle\right)= \begin{cases}\left\langle j_{1}, j_{2}\right\rangle, & \text { if } j_{1} \neq j_{2} \\ \Delta_{J}, & \text { if } j_{1}=j_{2}\end{cases}
$$

and let $F_{1}$ denote the composition

$$
X^{2} \stackrel{F_{0}}{\longrightarrow} J^{2} \stackrel{\Psi}{\rightarrow} J^{2,1}
$$

where $F_{0}: X^{2} \rightarrow J^{2}$ is defined by (2.13). Then $F_{1}$ belongs to $\operatorname{Coh}^{1}\left(R_{Q}\right)$ and $(2.16)$ holds.

Let $X$ be a nonempty set and let $Q$ be a partition of the set $X^{2}, Q \in \Pi\left(X^{2}\right)$. We say that $Q$ is symmetric if the equivalence

$$
(\langle x, y\rangle \in B) \Leftrightarrow(\langle y, x\rangle \in B)
$$

is valid for each block $B$ of $Q$ and every $\langle x, y\rangle \in X^{2}$. Thus $Q$ is a symmetric partition of $X^{2}$ if every block of $Q$ is a symmetric binary relation on $X$.

The following proposition shows that, for every $P \in \Pi(X)$, the partition $P \otimes P_{S}$ defined by (2.9) is the smallest symmetric partition of $X^{2}$ with refinement $P \otimes P$.

Proposition 2.20. Let $X$ be a nonempty set and let $P=\left\{X_{j}: j \in J\right\}$ be a partition of $X$. Then $P \otimes P_{S}$ is symmetric and the inequality

$$
P \otimes P \leqslant X^{2} P \otimes P_{S}
$$

holds and, moreover, if $Q$ is an arbitrary symmetric partition of $X^{2}$ such that

$$
P \otimes P \leqslant X^{2} Q
$$


then we also have

$$
P \otimes P_{S} \leqslant X^{2} Q
$$

Proof. It follows directly from the definition of $P \otimes P_{S}$ that (2.18) holds and $P \otimes P_{S}$ is symmetric.

Suppose $Q \in \boldsymbol{\Pi}\left(X^{2}\right)$ is symmetric and satisfies (2.19). Let $\langle x, y\rangle$ be an arbitrary point of $X^{2}$. Then there are $j_{1}, j_{2} \in J$ such that

$$
\langle x, y\rangle \in\left(X_{j_{1}} \times X_{j_{2}}\right) \cup\left(X_{j_{2}} \times X_{j_{1}}\right) .
$$

Similarly, there is a block $B$ of $Q$ such that

$$
\langle x, y\rangle \in B
$$

There is also a block of $P \otimes P$ which contains $\langle x, y\rangle$. Using (2.21) we can suppose, for definiteness, that this is the block $X_{j_{1}} \times X_{j_{2}}$,

$$
\langle x, y\rangle \in X_{j_{1}} \times X_{j_{2}} .
$$

The last membership relation, (2.22) and inequality (2.19) imply

$$
X_{j_{1}} \times X_{j_{2}} \subseteq B
$$

Now to prove (2.20) it suffices to show that

$$
\left(X_{j_{2}} \times X_{j_{1}}\right) \subseteq B
$$

For every binary relation $R \subseteq X \times X$ the converse relation $R^{-1}$ is defined as

$$
R^{-1}:=\{\langle y, x\rangle:\langle x, y\rangle \in R\} .
$$

A binary relation $R$ is symmetric if and only if $R=R^{-1}$. From (2.23) it follows that

$$
X_{j_{2}} \times X_{j_{1}}=\left(X_{j_{1}} \times X_{j_{2}}\right)^{-1} \subseteq B^{-1} .
$$

Since $B$ is symmetric, the equality $B=B^{-1}$ holds. The inclusion

$$
X_{j_{2}} \times X_{j_{1}} \subseteq B
$$

follows.

Let $X$ be a nonempty set. A mapping $\Phi$ with domain $X^{2}$ is symmetric if the equality $\Phi(x, y)=\Phi(y, x)$ holds for all $x, y \in X$.

Lemma 2.21. Let $X$ be a nonempty set and let $\Phi$ be a surjective mapping with domain $X^{2}$. Then the mapping $\Phi$ is symmetric if and only if $P_{\Phi^{-1}}$ is a symmetric partition of $X^{2}$.

Proof. It follows directly from the definitions. 
Let $R$ be an equivalence relation on $X$. Denote by $\operatorname{Coh}_{S}(R)$ the class of all symmetric mappings $\Phi \in \operatorname{Coh}(R)$. The following theorem characterizes $P \otimes P_{S}$ as the smallest element of the set of all partitions of $X^{2}$ generated by mappings from $\mathrm{Coh}_{S}\left(R_{P}\right)$.

Theorem 2.22. Let $X$ be a nonempty set and let $Q=\left\{X_{j}: j \in J\right\}$ be a partition of $X$. Then the inequality

$$
Q \otimes Q_{S} \leqslant X^{2} P_{F^{-1}}
$$

holds for every $F \in \operatorname{Coh}_{S}\left(R_{Q}\right)$ and, moreover, there is $F_{1} \in \operatorname{Coh}_{S}\left(R_{Q}\right)$ such that the equality

$$
Q \otimes Q_{S}=P_{F_{1}^{-1}}
$$

holds.

Proof. Let $F \in \operatorname{Coh}_{S}\left(R_{Q}\right)$. By Theorem 2.16, we have the inequality

$$
Q \otimes Q \leqslant X^{2} P_{F^{-1}}
$$

Since $F$ is a symmetric mapping, the partition $P_{F^{-1}}$ is symmetric by Lemma 2.21 . Using Proposition 2.20, we see that inequality (2.24) follows from (2.26).

Let

$$
J \times J_{S}:=\left\{\left\{\left\langle j_{1}, j_{2}\right\rangle,\left\langle j_{2}, j_{1}\right\rangle\right\}:\left\langle j_{1}, j_{2}\right\rangle \in \nabla_{J}\right\} \cup \Delta_{J},
$$

where $\nabla_{J}=J^{2} \backslash \Delta_{J}$. Let us consider the surjection $\Psi: J^{2} \rightarrow J \times J_{S}$ such that

$$
\Psi\left(j_{1}, j_{2}\right)= \begin{cases}\left\{\left\langle j_{1}, j_{1}\right\rangle\right\}, & \text { if } j_{1}=j_{2} \\ \left\{\left\langle j_{1}, j_{2}\right\rangle,\left\langle j_{2}, j_{1}\right\rangle\right\}, & \text { if } j_{1} \neq j_{2}\end{cases}
$$

Write $F_{1}$ for the composition

$$
X^{2} \stackrel{F_{0}}{\rightarrow} J^{2} \stackrel{\Psi}{\rightarrow} J \times J_{S},
$$

where $F_{0}$ is defined by (2.13). Then $F_{1}$ belongs to $\operatorname{Coh}_{S}\left(R_{Q}\right)$ and equality $(2.25)$ holds.

Let $X$ be a nonempty set and let $P=\left\{X_{j}: j \in J\right\}$ be a partition of $X$. Recall that $P \otimes P_{S}^{1}$ is a partition of $X^{2}$ with the blocks $B$ such that either $B=R_{P}$ or there are distinct $j_{1}, j_{2} \in J$ for which

$$
B=\left(X_{j_{1}} \times X_{j_{2}}\right) \cup\left(X_{j_{2}} \times X_{j_{1}}\right)
$$

holds.

The following proposition shows that $P \otimes P_{S}^{1}$ is the smallest symmetric partition with refinement $P \otimes P^{1}$. 
Proposition 2.23. Let $X$ be a nonempty set and let $P$ be a partition of $X$. Then $P \otimes P_{S}^{1}$ is symmetric and the inequality

$$
P \otimes P^{1} \leqslant X^{2} P \otimes P_{S}^{1}
$$

holds and, moreover, if $Q$ is an arbitrary symmetric partition of $X^{2}$ such that

$$
P \otimes P^{1} \leqslant X^{2} Q
$$

then we also have

$$
P \otimes P_{S}^{1} \leqslant X^{2} Q
$$

Proof. It follows directly from the definitions of $P \otimes P_{S}^{1}$ and $P \otimes P^{1}$ that $P \otimes P_{S}^{1}$ is symmetric and (2.29) holds.

Suppose $Q \in \Pi\left(X^{2}\right)$ is symmetric and satisfies (2.30). Then we evidently have

$$
P \otimes P \leqslant X^{2} Q
$$

By Proposition 2.20, inequality (2.32) implies the inequality

$$
P \otimes P_{S} \leqslant X^{2} Q
$$

Inequality (2.31) follows from (2.33) and (2.30) because every block of $P \otimes P_{S}^{1}$ is a block of $P \otimes P^{1}$ or a block of $P \otimes P_{S}$.

Let $X$ be a nonempty set and let $R$ be an equivalence relation on $X$. We will denote by $\operatorname{Coh}_{S}^{1}(R)$ the class of all symmetric mappings $\Phi \in \operatorname{Coh}(R)$ satisfying the equality

$$
\Phi(x, x)=\Phi(y, y)
$$

for all $x, y \in X$. It is clear that the equality

$$
\operatorname{Coh}_{S}^{1}(R)=\operatorname{Coh}_{S}(R) \cap \operatorname{Coh}^{1}(R)
$$

holds for every nonvoid $\mathrm{X}$ and every equivalence relation $R$ on $X$.

Theorem 2.24. Let $X$ be a nonempty set and let $Q=\left\{X_{j}: j \in J\right\}$ be a partition of $X$. Then the inequality

$$
Q \otimes Q_{S}^{1} \leqslant X^{2} P_{\Phi^{-1}}
$$

holds for every $\Phi \in \operatorname{Coh}_{S}^{1}\left(R_{Q}\right)$ and, moreover, there is $\Phi_{1} \in \operatorname{Coh}_{S}^{1}\left(R_{Q}\right)$ such that the equality

$$
Q \otimes Q_{S}^{1}=P_{\Phi_{1}^{-1}}
$$

holds. 
Proof. Let $\Phi \in \operatorname{Coh}_{S}^{1}\left(R_{P}\right)$. Then, by (2.34), we obtain that $P_{\Phi^{-1}}$ is a symmetric partition of $X^{2}$. Since $\Phi \in \operatorname{Coh}\left(R_{P}\right)$ holds, Theorem 2.19 implies

$$
Q \otimes Q^{1} \leqslant X^{2} P_{\Phi^{-1}}
$$

Inequality (2.35) follows from $\Phi \in \mathrm{Coh}_{S}\left(R_{Q}\right)$, Proposition 2.23 and (2.37).

Let us find $\Phi_{1} \in \operatorname{Coh}_{S}^{1}\left(R_{Q}\right)$ such that (2.36) holds. Similarly (2.17) and (2.27), we define $J \times J_{S}^{1} \in \Pi\left(X^{2}\right)$ as

$$
J \times J_{S}^{1}:=\nabla_{J} \cup\left\{\Delta_{J}\right\} .
$$

Let us consider the mapping $\Psi_{1}: J \times J_{S} \rightarrow J \times J_{S}^{1}$ defined such that

$$
\Psi_{1}(\langle j, j\rangle)=\left\{\Delta_{J}\right\} \quad \text { and } \quad \Psi_{1}\left(\left\{\left\langle j_{1}, j_{2}\right\rangle,\left\langle j_{2}, j_{1}\right\rangle\right\}\right)=\left\{\left\langle j_{1}, j_{2}\right\rangle,\left\langle j_{2}, j_{1}\right\rangle\right\}
$$

hold for every $j \in J$ and all distinct $j_{1}, j_{2} \in J$. Write $\Phi_{1}$ for the composition

$$
X^{2} \stackrel{F_{0}}{\longrightarrow} J^{2} \stackrel{\Psi}{\rightarrow} J \times J_{S} \stackrel{\Psi_{1}}{\longrightarrow} J \times J_{S}^{1},
$$

where $F_{0}$ and $\Psi$ are defined by (2.13) and (2.28), respectively. Then $\Phi_{1}$ belongs to $\operatorname{Coh}_{S}^{1}\left(R_{Q}\right)$ and satisfies equality $(2.36)$.

The results of the present section are quite elementary and should be known to experts in the theory of relations in one form or another. Note also that these results can be naturally generalized to the case of partitions of the set $X^{K}$ for arbitrary $K$ with $|K| \geqslant 2$. The partitions $P \otimes P, P \otimes P^{1}, P \otimes P_{S}$ and $P \otimes P_{S}^{1}$ of $X^{2}$ can also be described as Cartesian products of disjoint unions of complete graphs with interpretation of $R_{P}$-coherent mappings as homomorphisms of corresponding graphs. (See, for example, [20] and [17] for some results related to Cartesian products and, respectively, morphisms of graphs.)

The algebraic structure of the subsemigroups of $\mathcal{B}_{X}$ generated by $P \otimes P, P \otimes P^{1}$ and $P \otimes P_{S}, P \otimes P_{S}^{1}$ will be described in Section 3 and, respectively, Section 4 of the paper.

\section{Semigroups generated by finest partitions of Cartesian squares}

Let $(\mathcal{S}, *)$ be a semigroup. If $\mathcal{S}$ is a single-point set, $\mathcal{S}=\{e\}$, then we consider that $e$ is the identity element of $(\mathcal{S}, *)$. The usual convention says that $(\mathcal{S}, *)$ must have at least two elements to posses a zero (see, for example, [18]).

An element $i \in \mathcal{S}$ is an idempotent element of $(\mathcal{S}, *)$ if

$$
i^{2}=i * i=i \text {. }
$$

It is clear that the identity element $e$ and the zero $\theta$ are idempotents. We will say that $e$ and $\theta$ are the trivial idempotent elements. 
Definition 3.1. Let $(\mathcal{S}, \circ)$ and $(\mathcal{H}, *)$ be semigroups. A mapping $F: \mathcal{S} \rightarrow \mathcal{H}$ is a homomorphism if

$$
F\left(s_{1} \circ s_{2}\right)=F\left(s_{1}\right) * F\left(s_{2}\right)
$$

holds for all $s_{1}, s_{2} \in \mathcal{S}$. If a homomorphism is injective, then it is a monomorphism. The bijective homomorphisms are called the isomorphisms.

The semigroups $\mathcal{S}$ and $\mathcal{H}$ are isomorphic if there is an isomorphism $F: \mathcal{S} \rightarrow \mathcal{H}$.

Recall that, for every nonempty set $Q$ of binary relations on a set $X$, we denote by $\mathcal{S}_{Q}$ a subsemigroup of $\mathcal{B}_{X}$ having $Q$ as a set of generators. In particular, if $Q$ is a partition of $X^{2}$, then every block of $Q$ is a binary relation on $X$ so that we can consider the semigroup $\mathcal{S}_{Q}$.

Now let $P$ be a partition of $X$. Then $P \otimes P$ is a partition of $X^{2}$ and our first goal is to describe the algebraic structure of the semigroup $\mathcal{S}_{P \otimes P}$ up to isomorphism.

Theorem 3.2. Let $(\mathcal{H}, *)$ be a semigroup. The following two statements are equivalent.

( $i)$ There are a nonempty set $X$ and a partition $P$ of $X$ such that the semigroup $\left(\mathcal{S}_{P \otimes P}, \circ\right)$ is isomorphic to $(\mathcal{H}, *)$.

(ii) The semigroup $(\mathcal{H}, *)$ satisfies the following conditions.

$\left(i_{1}\right)(\mathcal{H}, *)$ contains a zero element $\theta$ if $|\mathcal{H}| \geqslant 2$.

$\left(i i_{2}\right)$ The equality

$$
x * y=\theta
$$

holds for all distinct idempotent elements $x, y \in \mathcal{H}$.

$\left(i i_{3}\right)$ If $i_{l}$ and $i_{r}$ are nontrivial idempotent elements of $\mathcal{H}$, then there is a unique nonzero $a \in \mathcal{H}$ such that

$$
a=i_{l} * a * i_{r} .
$$

(ii $\left.{ }_{4}\right)$ If $|\mathcal{H}| \geqslant 2$ holds, then for every nonzero $a \in \mathcal{H}$ there is a unique pair $\left(i_{l a}, i_{r a}\right)$ of nontrivial idempotent elements of $\mathcal{H}$ such that

$$
a=i_{l a} * a * i_{r a} .
$$

Proof. $(i) \Rightarrow\left(\right.$ ii) Let $P=\left\{X_{j}: j \in J\right\}$ be a partition of a set $X$ such that the semigroup $(\mathcal{H}, *)$ is isomorphic to $\left(\mathcal{S}_{P \otimes P}, \circ\right)$. We must prove that $(\mathcal{H}, *)$ satisfies conditions $\left(i i_{1}\right)-\left(i i_{4}\right)$. Since $(\mathcal{H}, *)$ and $\left(\mathcal{S}_{P \otimes P}, \circ\right)$ are isomorphic, it suffices to show that the similar conditions hold for $\left(\mathcal{S}_{P \otimes P}, \circ\right)$. Let us do it.

First of all we note that conditions $\left(i i_{1}\right)-\left(i i_{4}\right)$ are satisfied if $|P|=1$. Suppose $|P| \geqslant 2$ holds.

$\left(i i_{1}\right)$. The inequality $|P| \geqslant 2$ implies that there are two distinct $X_{j_{1}}, X_{j_{2}} \in P$. Then we have $X_{j_{1}} \times X_{j_{1}} \in P \otimes P$ and $X_{j_{2}} \times X_{j_{2}} \in P \otimes P$ and $X_{j_{1}} \cap X_{j_{2}}=\varnothing$. Consequently

$$
\mathcal{S}_{P \otimes P} \ni\left(X_{j_{1}} \times X_{j_{1}}\right) \circ\left(X_{j_{2}} \times X_{j_{2}}\right)=\varnothing
$$


Thus $\mathcal{S}_{P \otimes P}$ contains the empty binary relation. This is the zero element of $\left(\mathcal{S}_{P \otimes P}, \circ\right)$.

In order to verify the fulfillment of $\left(i i_{2}\right)-\left(i i_{4}\right)$, we note that

$$
\left(X_{j_{1}} \times X_{j_{2}}\right) \circ\left(X_{j_{3}} \times X_{j_{4}}\right)= \begin{cases}\varnothing, & \text { if } X_{j_{2}} \neq X_{j_{3}} \\ \left(X_{j_{1}} \times X_{j_{4}}\right), & \text { if } X_{j_{2}}=X_{j_{3}}\end{cases}
$$

holds for all $X_{j_{1}}, X_{j_{2}}, X_{j_{3}}, X_{j_{4}} \in P$. Thus every element of $\left(\mathcal{S}_{P \otimes P}, \circ\right)$ is either empty or belongs to $P \otimes P$.

$\left(i i_{2}\right)$. From (3.4) it follows that every nontrivial idempotent element $i$ of $\left(\mathcal{S}_{P \otimes P}, \circ\right)$ has the form $i=X_{j} \times X_{j}$ for some $X_{j} \in P$. Now $\left(i i_{2}\right)$ follows from the equality $X_{j_{1}} \cap X_{j_{2}}=\varnothing$ which holds for all different $X_{j_{1}}, X_{j_{2}} \in P$.

$\left(i i_{3}\right)$. Let $i_{l}$ and $i_{r}$ be nontrivial idempotent elements of $\left(\mathcal{S}_{P \otimes P}, \circ\right)$. Then there are $j_{1}, j_{2} \in J$ such that

$$
i_{l}:=X_{j_{1}} \times X_{j_{1}} \quad \text { and } \quad i_{r}:=X_{j_{2}} \times X_{j_{2}}
$$

It is clear that (3.2) holds if $a=X_{j_{1}} \times X_{j_{2}}$. Suppose now that there is a nonzero $b \in \mathcal{H}$ such that

$$
b=i_{l} * b * i_{r} .
$$

Then we can find $j_{3}, j_{4} \in J$ such that $b=X_{j_{3}} \times X_{j_{4}}$. This equality and (3.5) give us

$$
\begin{aligned}
b=\left(X_{j_{3}} \times X_{j_{3}}\right) * b *\left(X_{j_{4}} \times X_{j_{4}}\right) & \\
& =\left(X_{j_{1}} \times X_{j_{1}}\right) *\left(X_{j_{3}} \times X_{j_{3}}\right) * b *\left(X_{j_{4}} \times X_{j_{4}}\right) *\left(X_{j_{2}} \times X_{j_{2}}\right) \neq \varnothing .
\end{aligned}
$$

Using $\left(i i_{2}\right)$, we have $X_{j_{1}}=X_{j_{3}}$ and $X_{j_{2}}=X_{j_{4}}$.

$\left(i i_{4}\right)$. Let $a \in \mathcal{H}$ be nonzero. It was shown above that there are $X_{j_{1}}, X_{j_{2}} \in P$ such that

$$
a=X_{j_{1}} \times X_{j_{2}}
$$

Write

$$
i_{l}:=X_{j_{1}} \times X_{j_{1}} \quad \text { and } \quad i_{r}:=X_{j_{2}} \times X_{j_{2}}
$$

Now (3.3) follows from (3.4). The uniqueness of representation (3.3) can be proved as above.

$($ ii $) \Rightarrow(i)$ Let $(\mathcal{H}, *)$ satisfy condition $(i i)$. Let $E=E(\mathcal{H})$ be the set of all idempotent elements of $\mathcal{H}$ and let $P$ be the partition of $E$ on the one-point subsets of $E$,

$$
P=\{\{i\}: i \in E\}
$$

We claim that the semigroup $\left(\mathcal{S}_{P \otimes P}, \circ\right)$ is isomorphic to $(\mathcal{H}, *)$. Using Proposition 2.4 and formula (3.4) we see that every element of $\left(\mathcal{S}_{P \otimes P}, \circ\right)$ is either empty or 
has a form $s=\left\{i_{1}\right\} \times\left\{i_{2}\right\}$ for some $i_{1}, i_{2} \in E$. From the definition of the Cartesian product, we have the equality

$$
\left\{i_{1}\right\} \times\left\{i_{2}\right\}=\left\{\left\langle i_{1}, i_{2}\right\rangle\right\},
$$

thus

$$
s=\left\{\left\langle i_{1}, i_{2}\right\rangle\right\},
$$

holds, where $\left\langle i_{1}, i_{2}\right\rangle \in E \times E$. Conditions $\left(i i_{3}\right)$ and $\left(i i_{4}\right)$ imply that there is a bijection $F: \mathcal{S}_{P \otimes P} \rightarrow \mathcal{H}$ such that $F(\varnothing)=\theta$, where $\theta$ is the zero element of $(\mathcal{H}, *)$, and $F\left(\left\{\left\langle i_{1}, i_{2}\right\rangle\right\}\right)=x$, where $x$ is a unique nonzero element of $\mathcal{H}$ such that

$$
x=i_{1} * x * i_{2} .
$$

It suffices to show that $F: \mathcal{S}_{P \otimes P} \rightarrow \mathcal{H}$ is an isomorphism. Let $s_{1}$ and $s_{2}$ belong to $\mathcal{S}_{P \otimes P}$. We must show that

$$
F\left(s_{1}\right) * F\left(s_{2}\right)=F\left(s_{1} \circ s_{2}\right) .
$$

This equality is trivially valid if $s_{1}=\varnothing$ or $s_{2}=\varnothing$. Suppose now that $s_{1} \neq \varnothing \neq s_{2}$ but

$$
s_{1} \circ s_{2}=\varnothing
$$

holds. Using (3.6) we can find $i_{1,1}, i_{1,2}, i_{2,1}, i_{2,2} \in E$ such that

$$
s_{1}=\left\{\left\langle i_{1,1}, i_{1,2}\right\rangle\right\} \quad \text { and } s_{1}=\left\{\left\langle i_{2,1}, i_{2,2}\right\rangle\right\} .
$$

From (3.9) it follows that $i_{1,2} \neq i_{2,1}$. By $\left(i i_{3}\right)$, there are the unique nonzero $x_{1}$ and $x_{2} \in \mathcal{H}$ such that

$$
x_{1}=i_{1,1} * x_{1} * i_{1,2} \quad \text { and } \quad x_{2}=i_{2,1} * x_{2} * i_{2,2} .
$$

By definition of $F$, we have the equalities $F(\varnothing)=\theta$ and $F\left(s_{i}\right)=x_{i}$ for $i=1,2$. Now using (3.11), condition $\left(i i_{2}\right)$ and $i_{1,2} \neq i_{2,1}$ we obtain

$$
\begin{aligned}
F\left(s_{1}\right) * F\left(s_{2}\right)=x_{1} * x_{2}=i_{1,1} * x_{1} *\left(i_{1,2}\right. & \left.* i_{2,1}\right) * x_{2} * i_{2,2} \\
& =i_{1,1} * x_{1} * \theta * x_{2} * i_{2,2}=\theta=F(\varnothing) .
\end{aligned}
$$

Suppose now that $s_{1}, s_{2}, s_{1} \circ s_{2}$ are nonzero elements of $\left(\mathcal{S}_{P \times P}, \circ\right)$. Then (3.10) and (3.11) hold with $i_{1,2}=i_{2,1}$ and $s_{1} \circ s_{2}=\left\{\left\langle i_{1,1}, i_{2,2}\right\rangle\right\}$. It implies

$$
F\left(s_{1}\right) * F\left(s_{2}\right)=\left(i_{1,1} * x_{1} * i_{1,2}\right) *\left(i_{1,2} * x_{2} * i_{2,2}\right)=x_{1} * x_{2} .
$$

Since $i_{1,1}, i_{1,2}, i_{2,2}$ are idempotent, from (3.11) we have

$$
i_{1,1} * x_{1} * i_{1,2}=i_{1,1} * x_{1} \quad \text { and } \quad i_{2,1} * x_{1} * i_{2,2}=x_{1} * i_{2,2} .
$$

Now using (3.12) we obtain

$$
F\left(s_{1}\right) * F\left(s_{2}\right)=i_{1,1} *\left(x_{1} * x_{2}\right) * i_{2,2} .
$$


By definition of $F$, there is a unique nonzero $y \in \mathcal{H}$ such that

$$
F\left(\left\{\left\langle i_{1,1}, i_{2,2}\right\rangle\right\}\right)=y=i_{1,1} * y * i_{2,2} .
$$

Thus

$$
F\left(s_{1} \circ s_{2}\right)=F\left(s_{1}\right) * F\left(s_{2}\right)
$$

holds for all $s_{1}, s_{2} \in \mathcal{S}_{P \otimes P}$. The implication $(i i) \Rightarrow(i)$ follows.

Let us denote by $\mathbf{H}_{1}$ the class of all semigroups $(\mathcal{H}, *)$ satisfying conditions $\left(i i_{1}\right)-\left(i i_{4}\right)$ from Theorem 3.2.

Lemma 3.3. Let $X$ be a set and let $P$ be a partition of $X$ with $|P| \geqslant 2$. Then the equality

$$
\mathcal{S}_{P \otimes P}=\{\varnothing\} \cup P \otimes P
$$

holds.

Proof. It follows from formula (3.4).

Remark 3.4. Equality (3.14) does not hold if $|P|=1$. In this case we have $\mathcal{S}_{P \otimes P}=P \otimes P$.

Corollary 3.5. Every semigroup $(\mathcal{H}, *) \in \mathbf{H}_{1}$ admits a d-transitive monomorphism $\mathcal{H} \rightarrow \mathcal{B}_{X}$ with a suitable set $X$.

Proof. By Theorem 3.2 for every $(\mathcal{H}, *) \in \mathbf{H}_{1}$ there are $X$ and $P \in \mathbf{\Pi}(X)$ such that $(\mathcal{H}, *)$ and $\left(\mathcal{S}_{P \otimes P}, \circ\right)$ are isomorphic. From Lemma 3.3 and Definition 1.1 it follows that the identity mapping Id: $\mathcal{S}_{P \otimes P} \rightarrow \mathcal{B}_{X}, \operatorname{Id}(s)=s$ for every $s \in \mathcal{S}_{P \otimes P}$, is a $d$-transitive monomorphism for every $P \in \boldsymbol{\Pi}(X)$. Consequently, if $\Phi: \mathcal{H} \rightarrow \mathcal{S}_{P \otimes P}$ is an isomorphism, then the mapping

$$
\mathcal{H} \stackrel{\Phi}{\rightarrow} \mathcal{S}_{P \otimes P} \stackrel{\mathrm{Id}}{\rightarrow} \mathcal{B}_{X}
$$

is a $d$-transitive monomorphism.

Analyzing the proof of Theorem 3.2 and using Example 2.5 we obtain the next corollary.

Corollary 3.6. The following conditions are equivalent for every semigroup $\mathcal{H}$.

(i) $\mathcal{H} \in \mathbf{H}_{1}$.

(ii) There is a nonempty set $X$ such that $\mathcal{H}$ and $\mathcal{S}_{P_{\Delta{ }^{2}}}$ are isomorphic, where $P_{\Delta_{X^{2}}}$ is a partition of $X^{2}$ corresponding to the diagonal on $X^{2}$.

The last corollary claims that a semigroup $\mathcal{H}$ belongs to $\mathbf{H}_{1}$ if and only if there is a nonempty set $X$ such that $\mathcal{H}$ is generated by set of all single-point subsets of $X^{2}$. 
Corollary 3.7. Let $\mathcal{H} \in \mathbf{H}_{1}$ and $\mathcal{S} \in \mathbf{H}_{1}$ hold. Then $\mathcal{H}$ and $\mathcal{S}$ are isomorphic semigroups if and only if $|\mathcal{H}|=|\mathcal{S}|$.

Using Lemma 3.3 and Remark 3.4, we obtain the following.

Corollary 3.8. If $(\mathcal{H}, *) \in \mathbf{H}_{1}$ is finite, then $|\mathcal{H}| \neq 2$ and there is a nonnegative integer $n$ such that $|\mathcal{H}|=n^{2}+1$. Conversely, if $n \neq 1$ is a nonnegative integer, then there is $(\mathcal{H}, *) \in \mathbf{H}_{1}$ such that $|\mathcal{H}|=n^{2}+1$.

Condition $\left(i i_{2}\right)$ of Theorem 3.2 implies that, for every $(\mathcal{H}, *) \in \mathbf{H}_{1}$, the set $E=E(\mathcal{H})$ of all idempotent elements of $(\mathcal{H}, *)$ is a subsemigroup of $\mathcal{H}$. The following proposition shows that a $d$-transitive monomorphism $E(\mathcal{H}) \rightarrow \mathcal{B}_{X}$ exists if and only if $|\mathcal{H}|=1$.

Proposition 3.9. Let $(\mathcal{H}, *)$ be a semigroup with a zero element $\theta$. Suppose that all elements of $(\mathcal{H}, *)$ are idempotent, and the equality

$$
e_{1} * e_{2}=\theta
$$

holds for all distinct $e_{1}, e_{2} \in \mathcal{H}$. Then $(\mathcal{H}, *)$ does not admit any d-transitive monomorphism of the form $\mathcal{H} \rightarrow \mathcal{B}_{X}$.

Proof. Suppose contrary that there is a $d$-transitive monomorphism $\Phi: \mathcal{H} \rightarrow \mathcal{B}_{X}$.

Let $A$ be a set of generators of $(\mathcal{H}, *)$ such that $\{\Phi(a): a \in A\}$ is a partition of $X^{2}$. Let us define a subset $A_{1}$ of the set $A$ by the rule: a point $a \in A$ belongs to $A_{1}$ if and only if there is $x_{1} \in X$ such that

$$
\left\langle x_{1}, x_{1}\right\rangle \in \Phi(a) .
$$

We claim that the equality $A_{1}=A$ holds. Indeed, suppose the set $A \backslash A_{1}$ is nonempty. Let $b \in A \backslash A_{1}$. Then $\Phi(b)$ is a block of the partition $\{\Phi(a): a \in A\}$. Hence, $\Phi(b)$ is a nonempty subset of $X^{2}$. Let $x_{1}, x_{2}$ be points of $X$ such that

$$
\left\langle x_{1}, x_{2}\right\rangle \in \Phi(b) \text {. }
$$

Then there is $a_{1} \in A_{1}$ for which

$$
\left\langle x_{1}, x_{1}\right\rangle \in \Phi\left(a_{1}\right)
$$

holds. Since $A_{1} \cap\left(A \backslash A_{1}\right)=\varnothing$, we have $a_{1} \neq b$. From (3.15), it follows that $a_{1} * b=\theta$ and, by Definition 1.1,

$$
\Phi\left(a_{1} * b\right)=\Phi(\theta)=\varnothing .
$$

Since $\Phi$ is a homomorphism, $\Phi\left(a_{1} * b\right)=\Phi\left(a_{1}\right) \circ \Phi(b)$ holds. From (3.16) and (3.17) it follows that

$$
\left\langle x_{1}, x_{2}\right\rangle \in \Phi\left(a_{1}\right) \circ \Phi(b),
$$

that contradicts $(3.18)$. 
For every $a \in A$ define a subset $X_{a}$ of the set $X$ as

$$
X_{a}:=\{x \in X:\langle x, x\rangle \in \Phi(a)\} .
$$

Since $\{\Phi(a): a \in A\}$ is a partition of $X^{2}$ and for every $a \in A$ we have

$$
\Delta_{X} \cap \Phi(a) \neq \varnothing,
$$

the set $\left\{X_{a}: a \in A\right\}$ is a partition of $X$. Arguing as above, we see that the equality

$$
\Phi(a)=X_{a}^{2}
$$

holds for every $a \in A$. The equality

$$
X^{2}=\bigcup_{a \in A} \Phi(a)
$$

and (3.19) imply that

$$
X^{2}=\bigcup_{a \in A} X_{a}^{2}
$$

The last equality holds if and only if $|A|=1$. The set $A$ is a set of generators of $(\mathcal{H}, *)$. Consequently, we have $|\mathcal{H}|=1$, contrary to $\theta \in \mathcal{H}$.

Recall that a semigroup $(\mathcal{H}, *)$ is a group with zero if $(\mathcal{H}, *)$ contains a zero $\theta$ and the set $\mathcal{H} \backslash\{\theta\}$ is a group with respect to the multiplication $*$. The following proposition is almost evident.

Proposition 3.10. Let $(\mathcal{H}, *)$ be a group with zero. Then $(\mathcal{H}, *)$ does not admit any d-transitive monomorphism of the form $\mathcal{H} \rightarrow \mathcal{B}_{X}$.

Proof. Let $e$ be the identity of the group $\mathcal{H} \backslash\{\theta\}$. If $\Phi: \mathcal{H} \rightarrow \mathcal{B}_{X}$ is a $d$-transitive monomorphism with some nonempty set $X$, then $\Phi(\theta)=\varnothing$ and there is a set $A$ of generators of $(\mathcal{H}, *)$ such that $P=\{\Phi(a): a \in A\}$ is a partition of $X^{2}$. In particular, the equality

$$
\theta=a_{1} * \ldots * a_{n}
$$

holds with some $a_{1}, \ldots, a_{n} \in A$. Since every block $\Phi(a)$ of $P$ is nonempty subset of $X^{2}$, equality $\Phi(\theta)=\varnothing$ implies that $A$ is a subset of $\mathcal{H} \backslash\{\theta\}$. Hence, every $a \in A$ has an inverse element $a^{-1} \in \mathcal{H} \backslash\{\theta\}$. Using equality (3.20), we obtain

$$
\theta=a_{n}^{-1} * \ldots a_{1}^{-1} * \theta=\left(a_{n}^{-1} * \ldots a_{1}^{-1}\right) *\left(a_{1} * \ldots * a_{n}\right)=e .
$$

Thus, $\theta=e$ and, consequently, $\theta \in \mathcal{H} \backslash\{\theta\}$, contrary to the definition.

Corollary 3.5 and Proposition 3.9 show, in particular, that the existence of $d$ transitive monomorphism is not, in general, a hereditary property of semigroups. Now we consider an example of a semigroup for which this property is hereditary. Recall that a semigroup $(\mathcal{H}, *)$ is a right zero semigroup if $x * y=y$ holds for all $x, y \in \mathcal{H}$. The left zero semigroups are defined in a dual way (see, for example, $[6$, p. 4]). 
Proposition 3.11. Let $(\mathcal{H}, *)$ be a right (left) zero semigroup. Then every subsemigroup $\mathcal{S}$ of $(\mathcal{H}, *)$ admits a d-transitive monomorphism $\mathcal{S} \rightarrow \mathcal{B}_{X}$ for a suitable set $X$.

Proof. Suppose $(\mathcal{H}, *)$ is a right zero semigroup. Since every subsemigroup of $(\mathcal{H}, *)$ is also a right zero semigroup, it suffices to find a set $X$ and $d$-transitive monomorphism $\Phi: \mathcal{H} \rightarrow \mathcal{B}_{X}$. Write $X:=\mathcal{H}$ and

$$
P:=\{X \times\{x\}: x \in X\} .
$$

Then $P$ is a partition of $X^{2}$. Let us define a mapping $\Phi: \mathcal{H} \rightarrow \mathcal{B}_{X}$ as $\Phi(x)=X \times\{x\}$ for every $x \in \mathcal{H}$. Then the equalities

$$
\Phi(x * y)=\Phi(y)=X \times\{y\}
$$

and

$$
\Phi(x) \circ \Phi(y)=(X \times\{x\}) \circ(X \times\{y\})=X \times\{y\}
$$

hold for all $x, y \in \mathcal{H}$. Hence, $\Phi$ is a homomorphism. It is clear that $\Phi$ is injective and $\Phi(\mathcal{H})=P$. Since $\mathcal{H}$ is a set of generators of $(\mathcal{H}, *)$ and $\mathcal{H}$ has no zero element, the mapping $\Phi$ is $d$-transitive monomorphism.

For the case when $(\mathcal{H}, *)$ is a left zero semigroup it suffices to consider the partition $\{\{x\} \times X: x \in X\}$ instead of the partition $P$ defined by (3.21).

Example 3.12. Let $X=\{x \in \mathbb{R}: 0 \leqslant x \leqslant 1\}$ and let $P=\left\{X_{0}, X_{1}, X_{2}\right\}$ be a trichotomy of $X$ defined in Example 2.8. Write

$$
P^{r}:=\left\{X \times X_{0}, X \times X_{1}, X \times X_{2}\right\}
$$

and

$$
P^{l}:=\left\{X_{0} \times X, X_{1} \times X, X_{2} \times X\right\} .
$$

Then $P^{r}$ and $P^{l}$ are partitions of $X^{2}$ (see Figure 2), and $S_{P^{r}}$ is a right zero semigroup, and $S_{P^{l}}$ is a left zero semigroup.

To describe the algebraic structure of the semigroup $\mathcal{S}_{P \otimes P^{1}}$ (see (2.8)) we recall the procedure of "the adjunction of an identity element".

Let $(\mathcal{S}, *)$ be an arbitrary semigroup and let $\{e\}$ be a single-point set such that $e \notin \mathcal{S}$. We can extend the multiplication $*$ from $\mathcal{S}$ to $\mathcal{S} \cup\{e\}$ by the rule:

$$
e * e=e \quad \text { and } \quad e * x=x * e=x
$$

for every $x \in \mathcal{S}$. Following [6] we use the notation

$$
\mathcal{S}^{1}:= \begin{cases}\mathcal{S}, & \text { if }(\mathcal{S}, *) \text { has an identity element } \\ \mathcal{S} \cup\{e\}, & \text { otherwise. }\end{cases}
$$

It is clear that $e$ is an identity element of $\left(\mathcal{S}^{1}, *\right)$. Thus the semigroup $\left(\mathcal{S}^{1}, *\right)$ is obtained from $(\mathcal{S}, *)$ by "adjunction of an identity element to $(\mathcal{S}, *)$ ". 

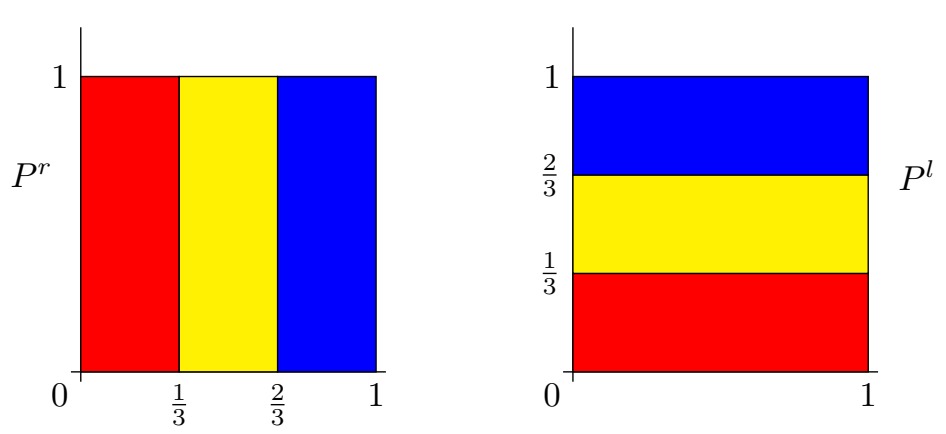

Figure 2. The partitions $P^{r}$ and $P^{l}$ corresponding to the trichotomy $\left\{X_{0}, X_{1}, X_{2}\right\}$. Here $X \times X_{0}\left(X_{0} \times X\right)$ is red, $X \times X_{1}$ $\left(X_{1} \times X\right)$ is yellow and $X \times X_{2}\left(X_{2} \times X\right)$ is blue.

Now we want to prove that for every nonempty set $X$ and every partition $P$ of $X$, the semigroup $\left(\mathcal{S}_{P \otimes P^{1}}, \circ\right)$ can be obtained from $\left(\mathcal{S}_{P \otimes P}, \circ\right)$ by adjunction of an identity element.

Lemma 3.13. Let $(\mathcal{H}, *)$ belong to $\mathbf{H}_{1}$. Then $(\mathcal{H}, *)$ contains an identity element if and only if $|\mathcal{H}|=1$.

Proof. If $|\mathcal{H}|=1$ holds, then $\mathcal{H}$ contains the identity element. Suppose now $|\mathcal{H}| \neq 1$. By condition $\left(i i_{1}\right)$ of Theorem $3.2,(\mathcal{H}, *)$ contains a zero element $\theta$. If $e$ is an identity element of $\mathcal{H}$, then $e \neq \theta$ holds. By condition $\left(i i_{4}\right)$ of Theorem 3.2, there exists a unique pair of idempotent elements $i_{l e}, i_{r e} \notin\{\theta, e\}$ such that

$$
e=i_{l e} * e * i_{r e}
$$

holds. Now we obtain

$$
i_{l e}=i_{l e} * e=i_{l e} *\left(i_{l e} * e * i_{r e}\right)=\left(i_{l e} * i_{l e}\right) * e * i_{r e}=i_{l e} * e * i_{r e}=e .
$$

Thus $i_{l e}=e$ holds contrary to $i_{l e} \notin\{\theta, e\}$.

Theorem 3.14. Let $(L, \cdot)$ be a semigroup. The following statements are equivalent.

(i) There are a set $X$ and a partition $P$ of $X$ such that the semigroup $\left(\mathcal{S}_{P \otimes P^{1}}, \circ\right)$ is isomorphic to $(L, \cdot)$.

(ii) There is a semigroup $(\mathcal{H}, *) \in \mathbf{H}_{1}$ such that $(L, \cdot)$ and $\left(\mathcal{H}^{1}, *\right)$ are isomorphic.

Tacking into account Theorem 3.2, we obtain the following equivalent reformulation of Theorem 3.14.

Theorem 3.15. Let $X$ be a set and let $P=\left\{X_{j}: j \in J\right\}$ be a partition of $X$. Then the semigroups $\left(\mathcal{S}_{P \otimes P}^{1}, \circ\right)$ and $\left(\mathcal{S}_{P \otimes P^{1}}, \circ\right)$ are isomorphic, where $\left(\mathcal{S}_{P \otimes P}^{1}, \circ\right)$ is obtained from $\left(\mathcal{S}_{P \otimes P}, \circ\right)$ by adjunction of an identity element. 
Proof. The case $|J|=1$ is trivial.

Let $|J| \geqslant 2$ hold. In this case, by Lemma $3.13,\left(\mathcal{S}_{P \otimes P}, \circ\right)$ does not contain any identity element. It is easy to see that

$$
P \otimes P^{-}:=\left\{X_{j_{1}} \times X_{j_{2}}:\left\langle j_{1}, j_{2}\right\rangle \in \nabla_{J}\right\},
$$

where $\nabla_{J}=J^{2} \backslash \Delta_{J}$, is a set of generators of the semigroup $\left(\mathcal{S}_{P \otimes P}, \circ\right)$. Indeed, the equality

$$
X_{j_{1}}^{2}=\left(X_{j_{1}} \times X_{j_{2}}\right) \circ\left(X_{j_{2}} \times X_{j_{1}}\right)
$$

holds for all $j_{1}, j_{2} \in J$. Hence, we have $P \otimes P \subseteq \mathcal{S}_{P \otimes P^{-}}$. Since $P \otimes P$ is a set of generators of $\left(\mathcal{S}_{P \otimes P}, \circ\right)$ and

$$
P \otimes P^{-} \subseteq P \otimes P
$$

holds, the equality $\left(\mathcal{S}_{P \otimes P^{-}}, \circ\right)=\left(\mathcal{S}_{P \otimes P}, \circ\right)$ follows.

For every $\left(X_{j_{1}} \times X_{j_{2}}\right) \in P \otimes P$ we evidently have

$$
\begin{aligned}
\left(X_{j_{1}} \times X_{j_{2}}\right) \circ R_{P} & =\left(X_{j_{1}} \times X_{j_{2}}\right) \circ\left(\bigcup_{j \in J} X_{j}^{2}\right) \\
& =\bigcup_{j \in J}\left(X_{j_{1}} \times X_{j_{2}}\right) \circ X_{j}^{2}=X_{j_{1}} \times X_{j_{2}}
\end{aligned}
$$

and, similarly,

$$
R_{P} \circ\left(X_{j_{1}} \times X_{j_{2}}\right)=X_{j_{1}} \times X_{j_{2}}
$$

holds. Moreover, we have

$$
\begin{aligned}
R_{P} \circ R_{P} & =\left(\bigcup_{j \in J} X_{j}^{2}\right) \circ\left(\bigcup_{j \in J} X_{j}^{2}\right) \\
& =\bigcup_{i, j \in J} X_{i}^{2} \circ X_{j}^{2}=\bigcup_{j \in J} X_{j}^{2}=R_{P}
\end{aligned}
$$

and $R_{P} \notin \mathcal{S}_{P \otimes P}$. Since $\left\{R_{P}\right\}=\mathcal{S}_{P \otimes P^{1}} \backslash \mathcal{S}_{P \otimes P}$ and $R_{P}$ is the identity element of $\left(\mathcal{S}_{P \otimes P^{1}}, \circ\right)$, the semigroup $\left(\mathcal{S}_{P \otimes P^{1}, \circ}\right)$ is obtained by adjunction of the identity element $R_{P}$ to $\left(\mathcal{S}_{P \otimes P}, \circ\right)$.

Corollary 3.16. Let $X$ be a nonempty set and let $P$ be a partition of $X$ with $|P| \geqslant 2$. Then we have

$$
\mathcal{S}_{P \otimes P^{1}}=\{\varnothing\} \cup\left\{R_{P}\right\} \cup P \otimes P .
$$

The proof of the next corollary is similar to the proof of Corollary 3.5.

Corollary 3.17. Let $(\mathcal{H}, *)$ belong to $\mathbf{H}_{1}$. Then $\left(\mathcal{H}^{1}, *\right)$ admits a d-transitive monomorphism $\mathcal{H}^{1} \rightarrow \mathcal{B}_{X}$ for a suitable set $X$. 


\section{Semigroups generated by finest symmetric partitions of Cartesian squares}

In what follows we say that a subsemigroup $\mathcal{H}_{1}$ of a semigroup $(\mathcal{H}, *)$ is an ideal of $\mathcal{H}$ if

$$
\mathcal{H}_{1} * \mathcal{H} \subseteq \mathcal{H}_{1} \quad \text { and } \quad \mathcal{H} * \mathcal{H}_{1} \subseteq \mathcal{H}_{1}
$$

holds, where we write

$$
A * B:=\{x * y: x \in A, y \in B\}
$$

for all nonempty subsets $A$ and $B$ of $\mathcal{H}$. An ideal $\mathcal{H}_{1}$ of a semigroup $\mathcal{H}$ is proper if $\left|\mathcal{H}_{1}\right|>1$ and $\mathcal{H} \neq \mathcal{H}_{1}$ hold.

Lemma 4.1. Let $\mathcal{C}$ be an ideal of a semigroup $(\mathcal{H}, *)$ and let $\theta$ be the zero of $\mathcal{C}$. Then $\theta$ is also the zero of $\mathcal{H}$.

Proof. Let $a$ belong to $\mathcal{H} \backslash \mathcal{C}$. Then $\theta * a$ belongs to $\mathcal{C}$ because $\theta \in \mathcal{C}$ and $\mathcal{C}$ is an ideal of $\mathcal{H}$. Consequently, we have

$$
\theta=\theta *(\theta * a)=(\theta * \theta) * a=\theta * a .
$$

Similarly we obtain $a * \theta=\theta$. Thus, $\theta$ is a zero of $(\mathcal{H}, *)$.

A semigroup is a band if every element of this semigroup is idempotent. (This notion was introduced in [22].) For every $(\mathcal{H}, *) \in \mathbf{H}_{1}$ the set $E=E(\mathcal{H})$ is a commutative band (this band was consider above in Proposition 3.9). A right (left) zero semigroup is an example of non-commutative band (see Proposition 3.11).

If the set $E(\mathcal{H})$ of all idempotent elements of a semigroup $\mathcal{H}$ is a band, then the set $E\left(\mathcal{H}^{1}\right)$ is also a band.

Example 4.2. Let $P=\left\{X_{j}: j \in J\right\}$ be a partition of a nonempty set $X$ and let $|J| \geqslant 2$. Then the sets $E\left(\mathcal{S}_{P \otimes P^{1}}\right)$ and $E\left(\mathcal{S}_{P \otimes P}\right)$ are commutative bands,

$$
E\left(\mathcal{S}_{P \otimes P^{1}}\right)=\{\varnothing\} \cup\left\{R_{P}\right\} \cup\left\{X_{j}^{2}: j \in J\right\}, \quad E\left(\mathcal{S}_{P \otimes P}\right)=\{\varnothing\} \cup\left\{X_{j}^{2}: j \in J\right\} .
$$

Every commutative band $(E, *)$ has a natural partial order $\leqslant$ defined by

$$
\left(i_{2} \leqslant i_{1}\right) \Leftrightarrow\left(i_{1} * i_{2}=i_{1}\right) .
$$

A colored Hasse diagram of $(E, \leqslant)$ is plotted in Figure 3 for the case when $E$ is the band of all idempotents of $\mathcal{S}_{P \otimes P^{1}}$ and $P=\left\{X_{0}, X_{1}, X_{2}\right\}$ is the trichotomy introduced in Example 2.8. A standard definition of the Hasse diagram for finite partially ordered sets can be found in [19, p. 15].

Definition 4.3. Let $(\mathcal{H}, *)$ be a semigroup and let $\mathcal{C}$ be an ideal of $(\mathcal{H}, *)$. The semigroup $(\mathcal{H}, *)$ is a band of subsemigroups with core $\mathcal{C}$ if there is a partition $\left\{\mathcal{H}_{\alpha}: \alpha \in \Omega\right\}$ of the set $\mathcal{H} \backslash \mathcal{C}$ such that every $\mathcal{H}_{\alpha}$ is a subsemigroup of $\mathcal{H}$ and $\mathcal{H}_{\alpha_{1}} * \mathcal{H}_{\alpha_{2}} \subseteq \mathcal{C}$ holds for all distinct $\alpha_{1}, \alpha_{2} \in \Omega$. 


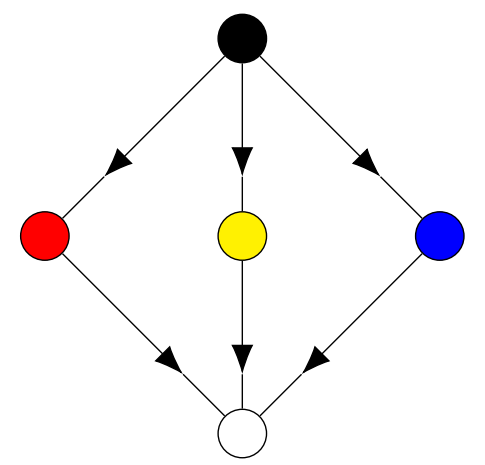

Figure $3 . R_{P}$ is white, $X_{0}^{2}$ is red, $X_{1}^{2}$ is yellow, $X_{2}^{2}$ is blue, and $\varnothing$ is black.

If $\mathcal{H}$ is a band of semigroups with core $\mathcal{C}$, then we write

$$
\mathcal{H} \approx\left\{\mathcal{H}_{\alpha}: \alpha \in \Omega\right\} \sqcup\{\mathcal{C}\} .
$$

Example 4.4. Let $\left\{\left(\mathcal{H}_{\alpha}, *_{\alpha}\right): \alpha \in \Omega\right\}$ be a nonempty set of disjoint semigroups and $(\mathcal{C}, \circ)$ be a semigroup with the zero element $\theta$ and such that

$$
\mathcal{C} \cap \mathcal{H}_{\alpha}=\varnothing
$$

holds for every $\alpha \in \Omega$. Let us define a binary operation $*$ on

$$
\mathcal{H}:=\mathcal{C} \cup\left(\bigcup_{\alpha \in \Omega} \mathcal{H}_{\alpha}\right)
$$

as

$$
x * y= \begin{cases}x \circ y, & \text { if } x, y \in \mathcal{C} \\ x *_{\alpha} y, & \text { if } x, y \in \mathcal{H}_{\alpha}, \alpha \in \Omega \\ \theta, & \text { otherwise. }\end{cases}
$$

It is easy to prove that $*$ is associative. Hence, $(\mathcal{H}, *)$ is a semigroup, and, in addition, from (4.3) it follows directly that

$$
\mathcal{H} \approx\left\{\mathcal{H}_{\alpha}: \alpha \in \Omega\right\} \sqcup\{\mathcal{C}\}
$$

Example 4.5. Let $(\mathcal{C}, \circ)$ and $(\mathcal{S}, \cdot)$ be disjoint semigroups and let $\mathcal{H}=\mathcal{C} \cup \mathcal{S}$. Write

$$
x * y= \begin{cases}x \circ y, & \text { if } x, y \in \mathcal{C} \\ x \cdot y, & \text { if } x, y \in \mathcal{S} \\ x, & \text { if } x \in \mathcal{C} \text { and } y \in \mathcal{S} \\ y, & \text { if } y \in \mathcal{C} \text { and } x \in \mathcal{S} .\end{cases}
$$

Then $*: \mathcal{H} \times \mathcal{H} \rightarrow \mathcal{H}$ is an associative operation, and $(\mathcal{H}, *)$ is a band of semigroups with core $\mathcal{C}$. 
The defined above band of subsemigroups with given core can be considered as a special case of the union of band of semigroups (see, for example, [6, p. 25]). Recall that a semigroup $(\mathcal{H}, *)$ is a union of band of subsemigroups $\mathcal{H}_{\alpha}, \alpha \in \Omega$ if

$$
P^{\mathcal{H}}:=\left\{\mathcal{H}_{\alpha}: \alpha \in \Omega\right\}
$$

is a partition of $\mathcal{H}$ and $\mathcal{H}_{\alpha} * \mathcal{H}_{\alpha} \subseteq \mathcal{H}_{\alpha}$ holds for every $\alpha \in \Omega$ and, moreover, for every pair of distinct $\alpha, \beta \in \Omega$ there is $\gamma \in \Omega$ such that $\mathcal{H}_{\alpha} * \mathcal{H}_{\beta} \subseteq \mathcal{H}_{\gamma}$.

The next theorem gives us a characterization of subsemigroups of $\mathcal{B}_{X}$ generated by partitions $P \otimes P_{S}$ of $X^{2}$ (see formula (2.9) and Proposition 2.20).

In what follows we denote by $\theta$ a zero element of $\operatorname{semigroup~}(\mathcal{H}, *)$.

Theorem 4.6. Let $(\mathcal{H}, *)$ be a semigroup and let $E=E(\mathcal{H})$ be the set of all idempotent elements of $(\mathcal{H}, *)$. Then the following conditions $(i)$ and $(i i)$ are equivalent.

(i) There is a nonempty set $X$ and a partition $P$ of $X$ such that $|P| \geqslant 2$ and the semigroup $(\mathcal{H}, *)$ is isomorphic to $\left(\mathcal{S}_{P \otimes P_{S}}, \circ\right)$.

(ii) The semigroup $(\mathcal{H}, *)$ is a band of semigroups $\mathcal{H}_{\alpha}$ with a core $\mathcal{C}$,

$$
\mathcal{H} \approx\left\{\mathcal{H}_{\alpha}: \alpha \in \Omega\right\} \sqcup\{\mathcal{C}\}
$$

and the following conditions hold.

(ii $\left.i_{1}\right) \mathcal{C}$ belongs to $\mathbf{H}_{1}$.

$\left(i i_{2}\right)$ Every $\mathcal{H}_{\alpha}$ is a group of order 2.

(ii $\left.i_{3}\right)$ is a commutative band.

$\left(i_{4}\right)$ If $e_{1}, e_{2}$ are two distinct nontrivial idempotent elements of $\mathcal{C}$, then there is a unique $e \in E \backslash \mathcal{C}$ such that

$$
e_{1}=e_{1} * e \text { and } e_{2}=e_{2} * e .
$$

Conversely, if $e \in E \backslash \mathcal{C}$, then there are exactly two distinct nontrivial $e_{1}, e_{2} \in \mathcal{C} \cap E$ such that (4.6) holds.

(ii $\left.i_{5}\right)$ For every $x \in E \cap \mathcal{C}$ and every $y \in \mathcal{H} \backslash E$ the equality $x * y=\theta$ $(y * x=\theta)$ holds if and only if $x * y(y * x)$ is idempotent.

Proof. $(i) \Rightarrow(i i)$ Let $P=\left\{X_{j}: j \in J\right\}$ be a partition of a set $X$ with $|P| \geqslant 2$ and let $\left(\mathcal{S}_{P \otimes P_{S}}, \circ\right)$ be isomorphic to $(\mathcal{H}, *)$. We must find a proper ideal $\mathcal{C}$ of $(\mathcal{H}, *)$ such that $\mathcal{C} \in \mathbf{H}_{1}$ and prove that $\mathcal{H}$ is a band with core $\mathcal{C}$ satisfying conditions $\left(i i_{2}\right)-\left(i i_{5}\right)$. Since $(\mathcal{H}, *)$ and $\left(\mathcal{S}_{P \otimes P_{S}}, \circ\right)$ are isomorphic and $\mathcal{S}_{P \otimes P}$ belongs to $\mathbf{H}_{1}$, it suffices to show that $\mathcal{S}_{P \otimes P_{S}}$ is a band with core $\mathcal{S}_{P \otimes P}$ and that conditions $\left(i i_{2}\right)-\left(i i_{5}\right)$ hold with $\mathcal{H}=\mathcal{S}_{P \otimes P_{S}}$ and $\mathcal{C}=\mathcal{S}_{P \otimes P}$

Suppose first $|J|=2$. Then we have $P=\left\{X_{1}, X_{2}\right\}$ and

$$
P \otimes P=\left\{X_{1}^{2}, X_{2}^{2}, X_{1} \times X_{2}, X_{2} \times X_{1}\right\}
$$

and

$$
P \otimes P_{S}=\left\{X_{1}^{2}, X_{2}^{2},\left(X_{1} \times X_{2}\right) \cup\left(X_{2} \times X_{1}\right)\right\}
$$


Write for short

$$
a_{1,1}=X_{1}^{2}, \quad a_{2,2}=X_{2}^{2}, \quad a_{1,2}=\left(X_{1} \times X_{2}\right) \cup\left(X_{2} \times X_{1}\right) .
$$

In this notation we obtain

$$
X_{1} \times X_{2}=a_{1,2} \circ a_{2,2}, X_{2} \times X_{1}=a_{1,2} \circ a_{1,1} \text { and } \varnothing=a_{1,1} \circ a_{2,2} .
$$

Lemma 3.3 and equalities (4.7) imply

$$
\mathcal{S}_{P \otimes P}=\left\{a_{1,1}, a_{2,2}, a_{1,2} \circ a_{2,2}, a_{1,2} \circ a_{1,1}, a_{1,1} \circ a_{2,2}\right\} .
$$

Thus $\mathcal{S}_{P \otimes P}$ is a subsemigroup of $\mathcal{S}_{P \otimes P_{S}}$. We notice that $P \otimes P_{S}$ is a set of generators of $\mathcal{S}_{P \otimes P_{S}}$, and $P \otimes P$ is a set of generators of $\mathcal{S}_{P \otimes P}$, and $a_{1,2}$ is the unique element of $\left(P \otimes P_{S}\right) \backslash(P \otimes P)$. Consequently, the subsemigroup $\mathcal{S}_{P \otimes P}$ of $\mathcal{S}_{P \otimes P_{S}}$ is an ideal of $\mathcal{S}_{P \otimes P_{S}}$ if and only if

$$
a_{1,2} \circ x \in \mathcal{S}_{P \otimes P} \quad \text { and } \quad x \circ a_{1,2} \in \mathcal{S}_{P \otimes P}
$$

hold for every $x \in P \otimes P$. If $x=a_{1,1}$ or $x=a_{2,2}$, then $a_{1,2} \circ x \in \mathcal{S}_{P \otimes P}$ follows from (4.8). If

$$
x=X_{2} \times X_{1}=a_{1,2} \circ a_{1,1},
$$

then, using the equality

$$
a_{1,2} \circ a_{1,2}=a_{1,1} \cup a_{2,2}
$$

we obtain

$$
\begin{aligned}
a_{1,2} \circ x=a_{1,2} \circ\left(a_{1,2} \circ a_{1,1}\right)=\left(a_{1,2} \circ a_{1,2}\right) \circ a_{1,1} \\
=\left(a_{1,1} \cup a_{2,2}\right) \circ a_{1,1}=a_{1,1},
\end{aligned}
$$

that implies $a_{1,2} \circ x \in \mathcal{S}_{P \otimes P}$. Thus $a_{1,2} \circ x \in \mathcal{S}_{P \otimes P}$ holds for every $x \in P \otimes P$. Analogously we can prove that $x \circ a_{1,2} \in \mathcal{S}_{P \otimes P}$ is valid for every $x \in P \otimes P$. Thus $\mathcal{S}_{P \otimes P}$ is an ideal of $\mathcal{S}_{P \otimes P_{S}}$. This ideal is proper because we have $a_{1,2} \in$ $\mathcal{S}_{P \otimes P_{S}} \backslash \mathcal{S}_{P \otimes P}$ and $\mathcal{S}_{P \otimes P}$ is single-point if and only if $|P|=1$.

A similar proof shows that for every $P=\left\{X_{j}: j \in J\right\}$ with $|J| \geqslant 3$ the semigroup $\mathcal{S}_{P \otimes P}$ is a proper ideal of $\mathcal{S}_{P \otimes P_{S}}$.

Let us prove that $\mathcal{S}_{P \otimes P_{S}}$ is a band with core $\mathcal{S}_{P \otimes P}$ and verify conditions $\left(i i_{2}\right)-$ $\left(i i_{5}\right)$.

Write for short

$$
a_{i, j}=\left(X_{i} \times X_{j}\right) \cup\left(X_{j} \times X_{i}\right)
$$

for all $i, j \in J$. Let $a$ belong to $\mathcal{S}_{P \otimes P_{S}} \backslash \mathcal{S}_{P \otimes P}$. Since $\mathcal{S}_{P \otimes P}$ is an ideal of $\mathcal{S}_{P \otimes P_{S}}$, the element $a$ has a form

$$
a=a_{i_{1}, j_{1}} \circ a_{i_{2}, j_{2}} \circ \ldots \circ a_{i_{n}, j_{n}},
$$

where $n$ is a positive integer number and

$$
a_{i_{k}, j_{k}} \in\left(P \otimes P_{S}\right) \backslash(P \otimes P)
$$


for every $k \in\{1, \ldots, n\}$. We claim that $a$ is an element of the cyclic semigroup

$$
\left\langle\left\langle a_{i_{1}, j_{1}}\right\rangle\right\rangle=\left\{a_{i_{1}, j_{1}}, a_{i_{1}, j_{1}}^{2}, a_{i_{1}, j_{1}}^{3}, \ldots\right\} .
$$

It is clear if $n=1$. Let us consider the case $n \geqslant 2$. Condition (4.11) implies $i_{k} \neq j_{k}$ for every $k \in\{1, \ldots, n\}$. It is easy to prove that

$$
a_{i_{1}, j_{1}} \circ a_{i_{2}, j_{2}}=\varnothing
$$

holds if $\left\{i_{1}, j_{1}\right\} \cap\left\{i_{2}, j_{2}\right\}=\varnothing$. Consequently, $a \in \mathcal{S}_{P \otimes P_{S}} \backslash \mathcal{S}_{P \otimes P}$ implies

$$
\left|\left\{i_{1}, j_{1}\right\} \cap\left\{i_{2}, j_{2}\right\}\right| \geqslant 1 .
$$

Let

$$
\left|\left\{i_{1}, j_{1}\right\} \cap\left\{i_{2}, j_{2}\right\}\right|=1 .
$$

Without loss of generality we can set $j_{1}=j_{2}$. Then

$$
i_{1} \neq j_{1} \neq i_{2} \neq i_{1}
$$

and

$$
\begin{aligned}
a_{i_{1}, j_{1}} \circ a_{i_{2}, j_{2}}= & a_{i_{1}, j_{1}} \circ a_{j_{1}, i_{2}} \\
& =\left(\left(X_{i_{1}} \times X_{j_{1}}\right) \circ\left(\left(X_{j_{1}} \times X_{i_{2}}\right) \cup\left(X_{i_{2}} \times X_{j_{1}}\right)\right)\right) \\
& \cup\left(\left(X_{j_{1}} \times X_{i_{1}}\right) \circ\left(\left(X_{j_{1}} \times X_{i_{2}}\right) \cup\left(X_{i_{2}} \times X_{j_{1}}\right)\right)\right) \\
& =\left(X_{i_{1}} \times X_{i_{2}}\right) \cup \varnothing=X_{i_{1}} \times X_{i_{2}}
\end{aligned}
$$

hold. Hence, if we have (4.13), then $a \in \mathcal{S}_{P \otimes P}$ holds, contrary to $a \in \mathcal{S}_{P \otimes P_{S}} \backslash \mathcal{S}_{P \otimes P}$. Let us consider the case when

$$
\left|\left\{i_{1}, j_{1}\right\} \cap\left\{i_{2}, j_{2}\right\}\right|=2 .
$$

The last equality holds if and only if $\left\{i_{1}, j_{1}\right\}=\left\{i_{2}, j_{2}\right\}$. In this case we obtain

$$
\begin{aligned}
& a_{i_{1}, j_{1}} \circ a_{i_{2}, j_{2}}=a_{i_{1}, j_{1}} \circ a_{i_{1}, j_{1}}=a_{i_{1}, j_{1}}^{2} \\
& \quad=\left(\left(X_{i_{1}} \times X_{j_{1}}\right) \cup\left(X_{j_{1}} \times X_{i_{1}}\right)\right) \circ\left(\left(X_{i_{1}} \times X_{j_{1}}\right) \cup\left(X_{j_{1}} \times X_{i_{1}}\right)\right)=X_{i_{1}}^{2} \cup X_{j_{1}}^{2} .
\end{aligned}
$$

From Lemma 3.3 it follows that

$$
a_{i_{1}, j_{1}} \notin \mathcal{S}_{P \otimes P} \quad \text { and } \quad a_{i_{1}, j_{1}}^{2} \notin \mathcal{S}_{P \otimes P}
$$

if $i_{1} \neq j_{1}$. Similarly, it can be shown that

$$
\left\langle i_{1}, j_{1}\right\rangle=\left\langle i_{3}, j_{3}\right\rangle, \quad \ldots, \quad\left\langle i_{1}, j_{1}\right\rangle=\left\langle i_{n}, j_{n}\right\rangle .
$$

The membership relation $a \in\left\langle\left\langle a_{i_{1}, j_{1}}\right\rangle\right\rangle$ follows.

It is easy to prove that every cyclic semigroup

$$
\left\langle\left\langle a_{i, j}\right\rangle\right\rangle=\left\{a_{i, j}, a_{i, j}^{2}, a_{i, j}^{3}, \ldots\right\}
$$


is a group of order 2. Indeed, we have

$$
a_{i, j}=\left(X_{i} \times X_{j}\right) \cup\left(X_{j} \times X_{i}\right) \neq X_{i}^{2} \cup X_{j}^{2}=a_{i, j}^{2}
$$

and

$$
\begin{aligned}
a_{i, j}^{3}=a_{i, j}^{2} \circ a_{i, j} \quad=\left(X_{i}^{2} \cup X_{j}^{2}\right) \circ\left(\left(X_{i} \times X_{j}\right) \cup\left(X_{j} \times X_{i}\right)\right) \\
=\left(X_{i} \times X_{j}\right) \cup\left(X_{j} \times X_{i}\right)=a_{i, j},
\end{aligned}
$$

i.e., $a_{i, j}^{3}=a_{i, j}$. The last equality implies

$$
\left(a_{i, j}^{2}\right)^{2}=a_{i, j}^{4}=a_{i, j}^{3} \circ a_{i, j}=a_{i, j} \circ a_{i, j}=a_{i, j}^{2} .
$$

Thus $\left\langle\left\langle a_{i, j}\right\rangle\right\rangle=\left\{a_{i, j}, a_{i, j}^{2}\right\}$ is a group of order 2 with the identity element $a_{i, j}^{2}$.

Suppose now that $x_{1}, x_{2} \in \mathcal{S}_{P \otimes P_{S}} \backslash \mathcal{S}_{P \otimes P}$. If there is $\langle i, j\rangle \in J^{2}, i \neq j$, such that $x_{1} \in\left\langle\left\langle a_{i, j}\right\rangle\right\rangle$ and $x_{2} \in\left\langle\left\langle a_{i, j}\right\rangle\right\rangle$, then $x_{1} \circ x_{2} \in\left\langle a_{i, j}\right\rangle$ holds because $\left\langle\left\langle a_{i, j}\right\rangle\right\rangle$ is a group. If we have

$$
x_{1} \in\left\langle\left\langle a_{i_{1}, j_{1}}\right\rangle\right\rangle \text { and } x_{2} \in\left\langle\left\langle a_{i_{2}, j_{2}}\right\rangle\right\rangle
$$

and $\left\{i_{1}, j_{1}\right\} \neq\left\{i_{2}, j_{2}\right\}$, then there are integer $m \geqslant 2$ and $n \geqslant 2$ such that

$$
x_{1} \circ x_{2}=a_{i_{1}, j_{1}}^{m} \circ a_{i_{2}, j_{2}}^{n}=a_{i_{1}, j_{1}}^{m-1} \circ\left(a_{i_{1}, j_{1}} \circ a_{i_{2}, j_{2}}\right) \circ a_{i_{2}, j_{2}}^{n-1} .
$$

If $\left|\left\{i_{1}, j_{1}\right\} \cap\left\{i_{2}, j_{2}\right\}\right|=0$, then $a_{i_{1}, j_{1}} \circ a_{i_{2}, j_{2}}=\varnothing$ holds and, moreover, if we have $\left|\left\{i_{1}, j_{1}\right\} \cap\left\{i_{2}, j_{2}\right\}\right|=1$, then, as in (4.12), (4.14), we obtain

$$
a_{i_{1}, j_{1}} \circ a_{i_{2}, j_{2}} \in \mathcal{S}_{P \otimes P}
$$

Since $\mathcal{S}_{P \otimes P}$ is an ideal of $\mathcal{S}_{P \otimes P_{S}}$, it follows that $x_{1} \circ x_{2} \in \mathcal{S}_{P \otimes P}$. Note now that equality $\left\langle\left\langle a_{i, j}\right\rangle\right\rangle=\left\{a_{i, j}, a_{i, j}^{2}\right\}$ implies

$$
\left\langle\left\langle a_{i_{1}, j_{1}}\right\rangle\right\rangle \cap\left\langle\left\langle a_{i_{2}, j_{2}}\right\rangle\right\rangle=\varnothing
$$

if $\left\{i_{1}, j_{1}\right\} \neq\left\{i_{2}, j_{2}\right\}$. Thus $\mathcal{S}_{P \otimes P_{S}}$ is a band of groups $\left\langle\left\langle a_{i, j}\right\rangle\right\rangle$ with core $\mathcal{S}_{P \otimes P}$ and condition $\left(i i_{2}\right)$ holds.

$\left(i i_{3}\right)$. In what follows we denote by $E=E\left(\mathcal{S}_{P \otimes P_{S}}\right)$ the set of all idempotent elements of $\mathcal{S}_{P \otimes P_{S}}$. It suffices to show that

$$
e_{1} \circ e_{2}=e_{2} \circ e_{1} \quad \text { and } \quad e_{1} \circ e_{2} \in E
$$

hold for all $e_{1}, e_{2} \in E$. First of all we note that (4.15) is trivially valid if $e_{1}=\varnothing$ or $e_{2}=\varnothing$ or if $e_{1}$ and $e_{2}$ are idempotent in $\mathcal{S}_{P \otimes P}$.

Let

$$
\varnothing \neq e_{1} \in \mathcal{S}_{P \otimes P} \cap E \text { and } \quad e_{2} \in\left(\mathcal{S}_{P \otimes P_{S}} \backslash \mathcal{S}_{P \otimes P}\right) \cap E .
$$

Then there are $j_{1}, j_{2}, j_{3} \in J$ such that $j_{2} \neq j_{3}$, and

$$
e_{1}=X_{j_{1}}^{2}, \quad \text { and } \quad e_{2}=X_{j_{2}}^{2} \cup X_{j_{3}}^{2} .
$$


Direct calculations show that

$$
e_{1} \circ e_{2}=e_{2} \circ e_{1}= \begin{cases}e_{1}, & \text { if } j_{1} \in\left\{j_{2}, j_{3}\right\} \\ \varnothing, & \text { if } j_{1} \notin\left\{j_{2}, j_{3}\right\}\end{cases}
$$

that implies (4.15). If we have

$$
\varnothing \neq e_{2} \in \mathcal{S}_{P \otimes P} \cap E \quad \text { and } \quad e_{1} \in\left(\mathcal{S}_{P \otimes P_{S}} \backslash \mathcal{S}_{P \otimes P}\right) \cap E
$$

then (4.15) is proved in a similar way.

Now let

$$
e_{1}, e_{2} \in\left(\mathcal{S}_{P \otimes P_{S}} \backslash \mathcal{S}_{P \otimes P}\right) \cap E .
$$

Then there are $i_{1}, j_{1}, i_{2}, j_{2} \in J$ such that $i_{1} \neq j_{1}$ and $i_{2} \neq j_{2}$ and

$$
e_{1}=X_{i_{1}}^{2} \cup X_{j_{1}}^{2} \quad \text { and } \quad e_{2}=X_{i_{2}}^{2} \cup X_{j_{2}}^{2} .
$$

Using these equalities we obtain

$$
e_{1} \circ e_{2}=e_{2} \circ e_{1}= \begin{cases}e_{1}, & \text { if }\left\{i_{1}, j_{1}\right\}=\left\{i_{2}, j_{2}\right\} \\ \varnothing, & \text { if }\left\{i_{1}, j_{1}\right\} \cap\left\{i_{2}, j_{2}\right\}=\varnothing \\ X_{j}^{2}, & \text { if } j \text { is a unique element of }\left\{i_{1}, j_{1}\right\} \cap\left\{i_{2}, j_{2}\right\} .\end{cases}
$$

The last equality also implies (4.15). Condition $\left(i i_{3}\right)$ follows.

$\left(i i_{4}\right)$. Let $e_{1}$ and $e_{2}$ be two distinct nontrivial idempotent elements of $\mathcal{S}_{P \otimes P}$. Then there are $j_{1}, j_{2} \in J$ such that

$$
e_{1}=X_{j_{1}}^{2} \quad \text { and } \quad e_{2}=X_{j_{2}}^{2}
$$

Using (4.17) we can show that

$$
e=X_{j_{1}}^{2} \cup X_{j_{2}}^{2}
$$

is a unique idempotent element of $\mathcal{S}_{P \otimes P_{S}} \backslash \mathcal{S}_{P \otimes P}$ for which (4.6) holds.

Conversely, if $e \in E \cap\left(\mathcal{S}_{P \otimes P_{S}} \backslash \mathcal{S}_{P \otimes P}\right)$, then there are distinct $j_{1}, j_{2} \in J$ such that (4.19) holds. For every nontrivial idempotent $e_{3} \in \mathcal{S}_{P \otimes P}$, the equalities $e_{3} \circ e=e_{3}$ and (4.19) imply $e_{3}=X_{j_{1}}^{2}$ or $e_{3}=X_{j_{2}}^{2}$, as required.

$\left(i i_{5}\right)$. We want to prove that

$$
(x \circ y=\varnothing) \Leftrightarrow(x \circ y \in E)
$$

and

$$
(y \circ x=\varnothing) \Leftrightarrow(y \circ x \in E)
$$

are valid for all $x \in E \cap \mathcal{C}$ and $y \in \mathcal{S}_{P \otimes P_{S}} \backslash E$.

Let us prove (4.20). The implication $(x \circ y=\varnothing) \Rightarrow(x \circ y \in E)$ is trivial. In particular, this implication is valid if $x=\varnothing$. If $x \in E \cap \mathcal{C}$, and $x \neq \varnothing$, and $y \in \mathcal{S}_{P \otimes P_{S}} \backslash E$, then there are $j_{1}, j_{2}, j_{3} \in J$ such that $j_{2} \neq j_{3}$, and

$$
x=X_{j_{1}}^{2}, \quad \text { and } \quad y=\left(X_{j_{2}} \times X_{j_{3}}\right) \cup\left(X_{j_{3}} \times X_{j_{2}}\right) .
$$


These equalities imply

$$
x \circ y= \begin{cases}\varnothing, & \text { if } j_{1} \neq\left\{j_{2}, j_{3}\right\} \\ X_{j_{2}} \times X_{j_{3}}, & \text { if } j_{1}=j_{2} \\ X_{j_{3}} \times X_{j_{2}}, & \text { if } j_{1}=j_{3} .\end{cases}
$$

Since every idempotent element of $\mathcal{C}$ is either trivial or has a form $e=X_{j}^{2}$ for some $j \in J$, we see that (4.22) implies the converse implication

$$
(x \circ y \in E) \Rightarrow(x \circ y=\varnothing) .
$$

Equivalence (4.20) is valid. The similar proof shows that (4.21) is also valid.

$($ ii $) \Rightarrow(i)$ Suppose $(\mathcal{H}, *)$ is a band of semigroups $\mathcal{H}_{\alpha}$ with core $\mathcal{C}$,

$$
\mathcal{H} \approx\left\{\mathcal{H}_{\alpha}: \alpha \in \Omega\right\} \sqcup\{\mathcal{C}\}
$$

such that $\mathcal{C} \in \mathbf{H}_{1}$ and conditions $\left(i i_{2}\right)-\left(i i_{5}\right)$ hold. By Theorem 3.2, there are a nonempty set $X$ and a partition $P=\left\{X_{j}: j \in J\right\}$ of $X$ such that $(\mathcal{C}, *)$ is isomorphic to $\left(\mathcal{S}_{P \otimes P}, \circ\right)$.

Let $\Phi: \mathcal{C} \rightarrow \mathcal{S}_{P \otimes P}$ be an isomorphism. We want to show that there is a continuation of $\Phi$ to an isomorphism $\Phi_{S}: \mathcal{H} \rightarrow \mathcal{S}_{P \otimes P_{S}}$ and that $|P| \geqslant 2$ holds. The construction of $\Phi_{S}$ will be carried out in two stages.

At the first stage, we will extend $\Phi$ to a monomorphism (injective homomorphism) $\Phi_{1}: \mathcal{C} \cup E \rightarrow \mathcal{S}_{P \otimes P_{S}}$. It should be noted here that $\mathcal{C} \cup E$ is also a band of semigroups with core $\mathcal{C}$ because we have

$$
\mathcal{H} \approx\left\{\mathcal{H}_{\alpha}: \alpha \in \Omega\right\} \sqcup\{\mathcal{C}\}
$$

and every group $\mathcal{H}_{\alpha}$ contains a unique idempotent element, and, for every $e \in \mathcal{H} \backslash \mathcal{C}$, there is a unique $\alpha \in \Omega$ such that $\{e\}$ is a subgroup of $\left(\mathcal{H}_{\alpha}, *\right)$.

In the second stage we will prove that the monomorphism $\Phi_{1}$ can be extended to an isomorphism $\Phi_{S}: \mathcal{H} \rightarrow \mathcal{S}_{P \otimes P_{S}}$.

Inequality $|P| \geqslant 2$. The core $\mathcal{C}$ has at least two nontrivial idempotent elements. Indeed, $\left\{\mathcal{H}_{\alpha}: \alpha \in \Omega\right\}$ is a partition of $\mathcal{H} \backslash \mathcal{C}$. Consequently, $\mathcal{H} \backslash \mathcal{C}$ and $\Omega$ are nonempty sets (see Remark 2.3). By condition $\left(i i_{2}\right)$, every $\mathcal{H}_{\alpha}$ is a group. The identity element of $\mathcal{H}_{\alpha}$ is an idempotent element of $\mathcal{H}$ belonging to $\mathcal{H} \backslash \mathcal{C}$. Using condition $\left(i i_{4}\right)$, we see that $\mathcal{C}$ contains at least two nontrivial idempotent elements as stated above. Since $\mathcal{C}$ and $\mathcal{S}_{P \otimes P}$ are isomorphic and all idempotent elements of $\mathcal{S}_{P \otimes P}$ are trivial if $|P|=1$, we have $|P| \geqslant 2$.

Monomorphism $\Phi_{1}: \mathcal{C} \cup E \rightarrow \mathcal{S}_{P \otimes P_{S}}$. By condition $\left(i i_{4}\right)$, for every $x \in E \backslash \mathcal{C}$, there are exactly two distinct nontrivial idempotent elements $x_{1}, x_{2} \in \mathcal{C}$ such that

$$
x_{1}=x_{1} * x \quad \text { and } \quad x_{2}=x_{2} * x .
$$


Let us define a mapping $\Phi_{1}: \mathcal{C} \cup E \rightarrow \mathcal{S}_{P \otimes P_{S}}$ as follows

$$
\Phi_{1}(x)= \begin{cases}\Phi(x), & \text { if } x \in \mathcal{C} \\ \Phi\left(x_{1}\right) \cup \Phi\left(x_{2}\right), & \text { if } x \in E \backslash \mathcal{C},\end{cases}
$$

where $x_{1}$ and $x_{2}$ are idempotent elements from (4.23). Note that if $z$ is a nontrivial idempotent element in $\mathcal{C}$, then there is a unique $j \in J$ such that $\Phi(z)=X_{j}^{2}$. Consequently, in (4.24) we have

$$
\Phi_{1}(x)=\Phi\left(x_{1}\right) \cup \Phi\left(x_{2}\right)=X_{j_{1}}^{2} \cup X_{j_{2}}^{2},
$$

where $X_{j_{1}}^{2}=\Phi\left(x_{1}\right)$ and $X_{j_{2}}^{2}=\Phi\left(x_{2}\right)$. Since

$$
\Phi(\mathcal{C})=\mathcal{S}_{P \otimes P} \subseteq \mathcal{S}_{P \otimes P_{S}}
$$

and, for every $x \in E \backslash \mathcal{C}$, we have

$$
\Phi_{1}(x) \in \mathcal{S}_{P \otimes P_{S}} \backslash \mathcal{S}_{P \otimes P},
$$

$\Phi_{1}$ really is a mapping from $\mathcal{C} \cup E$ to $\mathcal{S}_{P \otimes P_{S}}$.

The mapping $\Phi_{1}$ is injective because $\Phi$ is injective and because condition $\left(i i_{4}\right)$ and equalities (4.24)-(4.25) imply

$$
\Phi_{1}(x) \neq \Phi_{1}(y)
$$

for all different $x, y \in E \backslash \mathcal{C}$.

Note that

$$
\Phi_{1}(\mathcal{C} \cup E)=\mathcal{S}_{P \otimes P} \cup\left\{X_{j}^{2} \cup X_{i}^{2}: i, j \in J \text { and } i \neq j\right\} .
$$

Indeed, we evidently have $\Phi_{1}(\mathcal{C})=\Phi(\mathcal{C})=\mathcal{S}_{P \otimes P}$ and

$$
\Phi_{1}(E \backslash \mathcal{C}) \subseteq\left\{X_{j}^{2} \cup X_{i}^{2}: i, j \in J \text { and } i \neq j\right\} .
$$

Using condition $\left(i i_{4}\right)$, for any two distinct $x_{1}, x_{2} \in \mathcal{C} \cap E$, we can find $x \in E \backslash \mathcal{C}$ such that (4.23) holds. Since $\Phi: \mathcal{C} \rightarrow \mathcal{S}_{P \otimes P}$ is an isomorphism, we have the equality

$$
\Phi(E \cap \mathcal{C})=\left\{X_{j}^{2}: j \in J\right\} .
$$

Consequently, if $i, j \in J$ and $i \neq j$, then there is $x \in E \backslash \mathcal{C}$ such that

$$
\Phi_{1}(x)=\Phi\left(x_{1}\right) \cup \Phi\left(x_{2}\right)=X_{j}^{2} \cup X_{i}^{2} .
$$

Hence, the inclusion

$$
\Phi_{1}(E \backslash \mathcal{C}) \supseteq\left\{X_{j}^{2} \cup X_{i}^{2}: i, j \in J \text { and } i \neq j\right\}
$$

holds. The last inclusion, (4.27) and (4.28) imply (4.26).

The mapping $\Phi_{1}$ is a monomorphism if and only if

$$
\Phi_{1}(x * y)=\Phi_{1}(x) \circ \Phi_{1}(y)
$$


holds for all $x, y \in \mathcal{C} \cup E$. Since $\Phi_{1}$ is an extension of the isomorphism $\Phi$, equality (4.30) is trivial for $x, y \in \mathcal{C}$. In addition, we have

$$
\Phi_{1}(x * x)=\Phi_{1}(x) \circ \Phi_{1}(x)
$$

for every $x \in E \cup \mathcal{C}$ because, for $x \in E \backslash \mathcal{C}$, from (4.25) it follows that $\Phi_{1}(x)$ is an idempotent element of $\mathcal{S}_{P \otimes P_{S}}$. Consequently, it suffices to prove equality (4.30) in the following cases:

$$
\begin{aligned}
& x \in E \backslash \mathcal{C} \text { and } \quad y \in E \cap \mathcal{C}, \\
& x \in E \cap \mathcal{C} \text { and } y \in E \backslash \mathcal{C}, \\
& x \in E \backslash \mathcal{C} \text { and } y \in \mathcal{C} \backslash E, \\
& x \in \mathcal{C} \backslash E \text { and } y \in E \backslash \mathcal{C}, \\
& x, y \in E \backslash \mathcal{C} \text { and } \quad x \neq y .
\end{aligned}
$$

Before proceeding to the proof of equality (4.30) for cases (4.31)-(4.35), we also note that this equality holds if

$$
x=\theta \quad \text { or } \quad y=\theta,
$$

when $\theta$ is the zero of $\mathcal{C}$. To see it, we suppose that (4.36) holds. By Lemma 4.1, $\theta$ also is the zero of $(\mathcal{H}, *)$. Thus, we have $x * y=\theta$ that implies

$$
\Phi_{1}(x * y)=\Phi_{1}(\theta)=\Phi(\theta)=\varnothing .
$$

Since $\Phi: \mathcal{C} \rightarrow \mathcal{S}_{P \otimes P}$ is an isomorphism and $\Phi_{1}$ is an extension of $\Phi$, from (4.36) and $|P| \geqslant 2$ it follows that

$$
\Phi_{1}(x)=\varnothing \quad \text { or } \quad \Phi_{1}(y)=\varnothing .
$$

Consequently,

$$
\Phi_{1}(x) \circ \Phi_{1}(y)=\varnothing
$$

holds. Thus, (4.36) implies equality (4.30).

Further, in proving equality (4.30), we will always assume

$$
y \neq \theta
$$

and, for $x \in E \backslash \mathcal{C}$, we will set $\Phi_{1}(x)=X_{j_{1}}^{2} \cup X_{j_{2}}^{2}$, where $j_{1}$ and $j_{2}$ are distinct elements of $J$ such that

$$
x_{1}:=\Phi^{-1}\left(X_{j_{1}}^{2}\right), \quad x_{2}:=\Phi^{-1}\left(X_{j_{2}}^{2}\right), \quad x_{1}=x_{1} * x, \quad x_{2}=x_{2} * x
$$

(see $(4.23)-(4.25))$.

Case (4.31). Taking condition (4.39) into account, we can find $j_{3} \in J$ for which

$$
\Phi_{1}(y)=\Phi(y)=X_{j_{3}}^{2} .
$$


Hence, the equality

$$
\Phi_{1}(x) \circ \Phi_{1}(y)= \begin{cases}\varnothing, & \text { if } j_{3} \notin\left\{j_{1}, j_{2}\right\} \\ X_{j_{3}}^{2}, & \text { if } j_{3} \in\left\{j_{1}, j_{2}\right\}\end{cases}
$$

holds. If $y=x_{1}$ or $y=x_{2}$, then from (4.40) it follows that

$$
y=x_{1}=x_{1} * x=y * x
$$

or, respectively,

$$
y=x_{2}=x_{2} * x=y * x .
$$

Thus

$$
\Phi(y * x)=\Phi_{1}(y)=\Phi\left(\Phi^{-1}\left(X_{j_{3}}^{2}\right)\right)=X_{j_{3}}^{2}
$$

holds which implies (4.30).

Suppose now $x_{1} \neq y \neq x_{2}$. Since $(\mathcal{H}, *)$ is a band of semigroups with core $\mathcal{C}$ and $y \in \mathcal{C}$, we have $x * y \in \mathcal{C}$. Moreover, by condition $\left(i i_{3}\right)$, we have $x * y \in E$. Consequently, $x * y \in E \cap \mathcal{C}$ holds. If $x * y \neq y$, then, using condition $\left(i i_{2}\right)$ of Theorem 3.2, we obtain

$$
x * y=x * y^{2}=(x * y) * y=\theta .
$$

Consequently, we have

$$
\Phi_{1}(x * y)=\Phi(x * y)=\Phi(\theta)=\varnothing
$$

Using (4.41) and (4.43) we obtain (4.30).

Case (4.32). This case is completely similar to the previous one.

Case (4.33). From $y \in \mathcal{C} \backslash E$ and (4.39) it follows that there is a pair $\left\langle j_{3}, j_{4}\right\rangle \in J^{2}$ such that

$$
\Phi_{1}(y)=X_{j_{3}} \times X_{j_{4}} \quad \text { and } \quad j_{3} \neq j_{4}
$$

Now we obtain

$$
\begin{aligned}
\Phi_{1}(x) \circ \Phi_{1}(y) & =\left(X_{j_{1}}^{2} \cup X_{j_{2}}^{2}\right) \circ\left(X_{j_{3}} \times X_{j_{4}}\right) \\
& = \begin{cases}\varnothing, & \text { if } j_{3} \notin\left\{j_{1}, j_{2}\right\} \\
X_{j_{3}} \times X_{j_{4}}, & \text { if } j_{3} \in\left\{j_{1}, j_{2}\right\}\end{cases} \\
& = \begin{cases}\varnothing, & \text { if } j_{3} \notin\left\{j_{1}, j_{2}\right\} \\
\Phi_{1}(y), & \text { if } j_{3} \in\left\{j_{1}, j_{2}\right\} .\end{cases}
\end{aligned}
$$

From condition $\left(i i_{4}\right)$ of Theorem 3.2 , it follows that $j_{3} \in\left\{j_{1}, j_{2}\right\}$ holds if and only if

$$
x_{1} * y=y \quad \text { or } \quad x_{2} * y=y
$$


Suppose we have $x_{1} * y=y$. From $x_{1}=x_{1} * x$ and $\left(i i_{3}\right)$ it follows that $x * x_{1}=x_{1}$. Consequently,

$$
x * y=x *\left(x_{1} * y\right)=\left(x * x_{1}\right) * y=x_{1} * y=y .
$$

Thus,

$$
\Phi_{1}(x * y)=\Phi_{1}(y)=\Phi(y)=\Phi_{1}(x) \circ \Phi_{1}(y)
$$

holds if $x_{1} * y=y$. Analogously, we obtain (4.46) if $x_{2} * y=y$.

Suppose now $j_{3} \notin\left\{j_{1}, j_{2}\right\}$. Write

$$
x_{3}:=\Phi^{-1}\left(X_{j_{3}}^{2}\right) .
$$

Then $x_{3}$ is an idempotent element of $\mathcal{C}$, and $\theta \neq x_{3}$, and

$$
x_{1} \neq x_{3} \neq x_{2} \text {. }
$$

By condition $\left(i i_{3}\right)$, we have $x * x_{3} \in E$ and, in addition, $x * x_{3} \in \mathcal{C}$ because $\mathcal{C}$ is an ideal of $(\mathcal{H}, *)$. Since $E$ is a commutative band, we obtain also

$$
x_{3} * x \in \mathcal{C} \cap E .
$$

It is clear that

$$
\left(x_{3} * x\right) * x=x_{3} *(x * x)=x_{3} * x .
$$

Condition $\left(i i_{4}\right)$ and (4.48)-(4.49) imply that $x_{3} * x$ is a trivial idempotent element of $\mathcal{C}$. Since $(\mathcal{C}, *)$ and $\left(\mathcal{S}_{P \otimes P}, \circ\right)$ are isomorphic and $|P| \geqslant 2$ holds, $\mathcal{C}$ contains a zero $\theta$, and it is the unique trivial idempotent element of $(\mathcal{C}, *)$. It follows directly from (4.47) and (4.44) that $y=x_{3} * y$. Consequently,

$$
\Phi_{1}(x * y)=\Phi_{1}\left(x * x_{3} * y\right)=\Phi_{1}\left(\left(x * x_{3}\right) * y\right)=\Phi_{1}(\theta * y)=\Phi_{1}(\theta)=\varnothing .
$$

Equality (4.30) follows.

Case (4.34). This case is completely similar to (4.33).

Case (4.35). Using (4.24) and (4.35) we can find two distinct nontrivial $y_{1}$, $y_{2} \in \mathcal{C} \cap E$ and, consequently, two distinct $j_{3}, j_{4} \in J$ such that

$$
y_{1}=y_{1} * y, \quad y_{2}=y_{2} * y, \quad\left\{j_{1}, j_{2}\right\} \neq\left\{j_{3}, j_{4}\right\}
$$

and

$$
\Phi_{1}(y)=\Phi_{1}\left(y_{1}\right) \cup \Phi_{1}\left(y_{2}\right), \quad \Phi_{1}\left(y_{1}\right)=X_{j_{3}}^{2}, \quad \Phi_{1}\left(y_{2}\right)=X_{j_{4}}^{2} .
$$

It should be noted here that $\left\{j_{1}, j_{2}\right\} \neq\left\{j_{3}, j_{4}\right\}$ holds because $\Phi_{1}$ is injective and, by (4.35), we have $x \neq y$. From (4.51) it follows that $\Phi_{1}(y)=X_{j_{3}}^{2} \cup X_{j_{4}}^{2}$. The last equality and (4.25) imply

$$
\begin{aligned}
\Phi_{1}(x) \circ \Phi_{1}(y)=( & \left.X_{j_{1}}^{2} \cup X_{j_{2}}^{2}\right) \circ\left(X_{j_{3}}^{2} \cup X_{j_{4}}^{2}\right) \\
= & \begin{cases}\varnothing, & \text { if }\left\{j_{1}, j_{2}\right\} \cap\left\{j_{3}, j_{4}\right\}=\varnothing \\
X_{j_{0}}^{2}, & \text { if } j_{0} \text { is a unique point of }\left\{j_{1}, j_{2}\right\} \cap\left\{j_{3}, j_{4}\right\} .\end{cases}
\end{aligned}
$$


Let $\left\{j_{1}, j_{2}\right\} \cap\left\{j_{3}, j_{4}\right\} \neq \varnothing$. Without loss of generality, we can set

$$
j_{0}=j_{1}=j_{3},
$$

or, an equivalent form,

$$
x_{1}=y_{1}=y_{0},
$$

where $y_{0}=\Phi^{-1}\left(X_{j_{0}}^{2}\right)$. Equality (4.30) evidently holds if

$$
x * y=y_{0} .
$$

Let us prove the last equality. From (4.40), (4.50), (4.51), and (4.53) it follows that

$$
y_{0}=y_{0} * x \quad \text { and } \quad y_{0}=y_{0} * y .
$$

These equalities and condition $\left(i i_{3}\right)$ imply

$$
y_{0}=y_{0}^{2}=\left(y_{0} * x\right) *\left(y_{0} * y\right)=\left(y_{0} * y_{0}\right) *(x * y)=y_{0} *(x * y) .
$$

Hence, $y_{0}=y_{0} *(x * y)$ holds. Since $(\mathcal{H}, *)$ is a band of $\mathcal{H}_{\alpha}$ with core $\mathcal{C}$ and $E$ is a commutative band, $x * y$ is an idempotent element of $\mathcal{C}$. Using condition $\left(i i_{2}\right)$ of Theorem 3.2, we see that $y_{0}=y_{0} *(x * y)$ holds if and only if $y_{0}=\theta$ or $y_{0}=x * y$. Since $\Phi\left(y_{0}\right)=X_{0}^{2} \neq \varnothing$, the equality $y_{0}=x * y$ holds.

Suppose now that $\left\{j_{1}, j_{2}\right\} \cap\left\{j_{3}, j_{4}\right\}=\varnothing$, i.e.,

$$
\left\{x_{1}, x_{2}\right\} \cap\left\{y_{1}, y_{2}\right\}=\varnothing .
$$

It suffices to show that $x * y=\theta$. As above, we can prove the membership relation $x * y \in E \cap \mathcal{C}$. Suppose that $x * y \neq \theta$, i.e., $z:=x * y$ is a nontrivial idempotent element of $\mathcal{C}$. It implies

$$
\theta \neq z=z *(x * y)=z^{2} *(x * y)=(z * x) *(z * y) .
$$

By condition $\left(i i_{2}\right)$ of Theorem 3.2, from (4.55) it follows that

$$
\theta \neq z=z * x \text { and } \quad \theta \neq z=z * y .
$$

Consequently, we have $z \in\left\{x_{1}, x_{2}\right\} \cap\left\{y_{1}, y_{2}\right\}$, contrary to (4.54).

Thus, $\Phi_{1}: \mathcal{C} \cup E \rightarrow \mathcal{S}_{P \otimes P_{S}}$ is a monomorphism.

Isomorphism $\Phi_{S}: \mathcal{H} \rightarrow \mathcal{S}_{P \otimes P_{S}}$. Let $x \in \mathcal{H} \backslash(\mathcal{C} \cup E)$. Then there is a unique $\alpha \in \Omega$ such that $x \in \mathcal{H}_{\alpha}$. Write $e_{x}=e_{\alpha}$ for the identity element of $\mathcal{H}_{\alpha}$. Then $e_{x} \in E \backslash \mathcal{C}$ holds and, by (4.25), we have

$$
\Phi_{1}\left(e_{x}\right)=X_{i}^{2} \cup X_{j}^{2},
$$

where $i=i(x)$ and $j=j(x)$ are some distinct elements of $J$. Let us define a mapping $\Phi_{S}: \mathcal{H} \rightarrow \mathcal{S}_{P \otimes P_{S}}$ as

$$
\Phi_{S}(x)= \begin{cases}\Phi_{1}(x), & \text { if } x \in E \cup \mathcal{C} \\ \left(X_{i} \times X_{j}\right) \cup\left(X_{j} \times X_{i}\right), & \text { if } x \in \mathcal{H} \backslash(E \cup \mathcal{C}),\end{cases}
$$


where $i=i(x)$ and $j=j(x)$ are elements of $J$ for which (4.56) holds. The mapping $\Phi_{S}$ is correctly defined because $\Phi_{1}$ is a mapping from $E \cup \mathcal{C}$ to $\mathcal{S}_{P \otimes P_{S}}$, and $\left\{\mathcal{H}_{\alpha}: \alpha \in\right.$ $\Omega\}$ is a partition of $\mathcal{H} \backslash \mathcal{C}$, and every $\mathcal{H}_{\alpha}$ is a group, and every group contains a unique identity element.

We claim that $\Phi_{S}$ is a bijection. Indeed, as in the proof of (4.29), we can show that for any two distinct $i, j \in J$ there is $\alpha \in \Omega$ such that the equality

$$
\Phi_{1}\left(e_{\alpha}\right)=X_{i}^{2} \cup X_{j}^{2}
$$

holds. If $x \neq e_{\alpha}$ and $x \in \mathcal{H}_{\alpha}$ hold, then from (4.57) and (4.58) we obtain

$$
\Phi_{S}(x)=\left(X_{i} \times X_{j}\right) \cup\left(X_{j} \times X_{i}\right) .
$$

Since we have the equality

$$
\begin{aligned}
\mathcal{S}_{P \otimes P_{S}}=\mathcal{S}_{P \otimes P} \cup\left\{\left(X_{i} \times X_{j}\right) \cup\left(X_{j} \times X_{i}\right): i, j \in J, i \neq j\right\} & \\
& \cup\left\{X_{i}^{2} \cup X_{j}^{2}: i, j \in J, i \neq j\right\},
\end{aligned}
$$

equality (4.26) implies that the mapping $\Phi_{S}$ is surjective. Moreover, $\left\{X_{j}: j \in J\right\}$ is a partition of $X$,

$$
\begin{aligned}
\left(X_{i_{1}}^{2} \cup X_{j_{1}}^{2}=X_{i_{2}}^{2} \cup X_{j_{2}}^{2}\right) \Leftrightarrow\left(\left\{i_{1}, j_{1}\right\}=\left\{i_{2}, j_{2}\right\}\right) & \\
& \Leftrightarrow\left(\left(X_{i_{1}} \times X_{j_{1}}\right) \cup\left(X_{j_{1}} \times X_{i_{1}}\right)=\left(X_{i_{2}} \times X_{j_{2}}\right) \cup\left(X_{j_{2}} \times X_{i_{2}}\right)\right)
\end{aligned}
$$

are valid for all two-point subsets $\left\{i_{1}, j_{1}\right\}$ and $\left\{i_{2}, j_{2}\right\}$ of $J$. Hence, $\Phi_{S}$ is injective and, consequently, bijective as was claimed above.

The bijection $\Phi_{S}: \mathcal{H} \rightarrow \mathcal{S}_{P \otimes P_{S}}$ is an isomorphism if and only if

$$
\Phi_{S}(x * y)=\Phi_{S}(x) \circ \Phi_{S}(y)
$$

holds for all $x, y \in \mathcal{H}$.

Let us prove equality (4.59).

First of all we note that (4.59) is equivalent to equality (4.30) if $x, y \in E \cup \mathcal{C}$. Moreover, if we have (4.36), then (4.59) can be proved similarly to (4.37)-(4.38).

In what follows we assume that (4.39) holds.

Suppose (4.59) holds if

$$
x \in \mathcal{C} \quad \text { and } \quad y \in \mathcal{H} \backslash(E \cup \mathcal{C})
$$

or if

$$
x \in \mathcal{H} \backslash(E \cup \mathcal{C}) \quad \text { and } \quad y \in \mathcal{C} .
$$

Then equality (4.59) holds for all $x, y \in \mathcal{H}$ if and only if it holds for all $x, y \in \mathcal{H} \backslash \mathcal{C}$. Let $t_{1}$ and $t_{2}$ be arbitrary points of $\mathcal{H} \backslash \mathcal{C}$. Then there are $\alpha_{1}, \alpha_{2} \in \Omega$ such that 
$t_{1} \in \mathcal{H}_{\alpha_{1}}$ and $t_{2} \in \mathcal{H}_{\alpha_{2}}$. Note that, for every $\alpha \in \Omega$, the restriction $\left.\Phi_{S}\right|_{\mathcal{H}_{\alpha}}: \mathcal{H}_{\alpha} \rightarrow$ $\Phi_{S}\left(\mathcal{H}_{\alpha}\right)$ is an isomorphism. Consequently, if $\alpha_{1}=\alpha_{2}$, then we have the equality

$$
\Phi_{S}\left(t_{1} * t_{2}\right)=\Phi_{S}\left(t_{1}\right) \circ \Phi_{S}\left(t_{2}\right) .
$$

In particular, we have

$$
\Phi_{S}\left(t * t^{2}\right)=\Phi_{S}\left(t^{2} * t\right)=\Phi_{S}(t) \circ \Phi_{S}\left(t^{2}\right)=\Phi_{S}\left(t^{2}\right) \circ \Phi_{S}(t)
$$

for every $t \in \mathcal{H}_{\alpha}$ and every $\alpha \in \Omega$.

Suppose $\alpha_{1} \neq \alpha_{2}$. Since every $\mathcal{H}_{\alpha}$ is a group of order 2 , the equalities

$$
t_{1}=t_{1}^{3} \quad \text { and } \quad t_{2}=t_{2}^{3}
$$

hold. Hence, we have

$$
\Phi_{S}\left(t_{1} * t_{2}\right)=\Phi_{S}\left(t_{1}^{3} * t_{2}^{3}\right)=\Phi_{S}\left(t_{1} *\left(t_{1}^{2} * t_{2}^{2}\right) * t_{2}\right) .
$$

Since $\mathcal{C}$ is a core of $\mathcal{H}$, the condition $\alpha_{1} \neq \alpha_{2}$ implies $t_{1}^{3} * t_{2}^{2} \in \mathcal{C}$, and $t_{1}^{2} * t_{2}^{3} \in \mathcal{C}$, and $t_{1}^{2} * t_{2}^{2} \in \mathcal{C}$. Consequently, from (4.63) and our supposition it follows that

$$
\begin{aligned}
\Phi_{S}\left(t_{1} * t_{2}\right)=\Phi_{S}\left(t_{1} *\left(t_{1}^{2} * t_{2}^{2}\right)\right) \circ \Phi_{S}\left(t_{2}\right) & \\
& =\Phi_{S}\left(t_{1}\right) \circ \Phi_{S}\left(t_{1}^{2} * t_{2}^{2}\right) \circ \Phi_{S}\left(t_{2}\right) .
\end{aligned}
$$

The elements $t_{1}^{2}, t_{2}^{2}$, and $t_{1}^{2} * t_{2}^{2}$ are idempotent and, by definition of $\Phi_{S}$, we have

$$
\left.\Phi_{1}\right|_{E}=\left.\Phi_{S}\right|_{E}
$$

Hence, using (4.62), (4.64), and (4.30), we obtain

$$
\begin{aligned}
\Phi_{S}\left(t_{1} * t_{2}\right)=\left(\Phi_{S}\left(t_{1}\right) \circ \Phi_{S}\left(t_{1}^{2}\right)\right) \circ\left(\Phi_{S}\left(t_{2}^{2}\right)\right. & \left.\circ \Phi_{S}\left(t_{2}\right)\right) \\
& =\Phi_{S}\left(t_{1}^{3}\right) \circ \Phi_{S}\left(t_{2}^{3}\right)=\Phi_{S}\left(t_{1}\right) \circ \Phi_{S}\left(t_{2}\right) .
\end{aligned}
$$

Consequently, it suffices to prove (4.59) if (4.60) or (4.61) holds. Notice now that instead of condition (4.60), we can use the stronger condition

$$
x \in \mathcal{C} \cap E \quad \text { and } \quad y \in \mathcal{H} \backslash(E \cup \mathcal{C}) .
$$

Indeed, from $\left(i i_{4}\right)$ of Theorem 3.2 it follows that for every $x \in \mathcal{C}$ there is a nontrivial idempotent $e \in \mathcal{C}$ such that

$$
x=x * e .
$$

For $x \in \mathcal{C}$ and $y \in \mathcal{H} \backslash(E \cup \mathcal{C})$, equality (4.66) implies

$$
\Phi_{S}(x * y)=\Phi_{S}(x * e * y)=\Phi_{S}((x * e) *(e * y)) .
$$

Since $x * e$ and $e * y$ belong to $\mathcal{C}$, we can rewrite (4.67) as

$$
\Phi_{S}(x * y)=\Phi_{S}(x * e) \circ \Phi_{S}(e * y)=\Phi_{S}(x) \circ \Phi_{S}(e) \circ \Phi_{S}(e * y) .
$$


It is clear that $e \in \mathcal{C} \cap E$. Consequently, if (4.59) holds for all $x, y$ satisfying (4.65), then (4.68) and (4.66) imply

$$
\begin{aligned}
\Phi_{S}(x * y)=\Phi_{S}(x) & \circ \Phi_{S}(e) \circ \Phi_{S}(e) \circ \Phi_{S}(y) \\
= & \Phi_{S}(x * e * e) \circ \Phi_{S}(y)=\Phi_{S}(x * e) \circ \Phi_{S}(y)=\Phi_{S}(x) \circ \Phi_{S}(y) .
\end{aligned}
$$

Similarly, instead of (4.61) we may use the condition

$$
x \in \mathcal{H} \backslash(E \cup \mathcal{C}) \text { and } y \in \mathcal{C} \cap E .
$$

Let (4.65) hold. Then, using (4.57) and (4.39), we can find $i, j, k \in J$ such that $i \neq j$ and

$$
\Phi_{S}(x)=X_{k}^{2} \quad \text { and } \quad \Phi_{S}(y)=\left(X_{i} \times X_{j}\right) \cup\left(X_{j} \times X_{i}\right) .
$$

From (4.70) it follows that

$$
\Phi_{S}(x) \circ \Phi_{S}(y)= \begin{cases}\varnothing, & \text { if } k \notin\{i, j\} \\ X_{j} \times X_{i}, & \text { if } k=j \\ X_{i} \times X_{j}, & \text { if } k=i .\end{cases}
$$

If $k \notin\{i, j\}$, then, using the equality $y^{3}=y$ and Lemma 4.1 as in (4.42), we obtain

$$
x * y=\left(x * y^{2}\right) * y=\theta * y=\theta,
$$

and, consequently,

$$
\Phi_{S}(x * y)=\Phi_{S}(\theta)=\varnothing .
$$

Now (4.59) follows from (4.71) and (4.72).

Let $k=j$ hold. Write

$$
e_{j}:=\Phi_{1}^{-1}\left(X_{j}^{2}\right) \quad \text { and } \quad e_{i}:=\Phi_{1}^{-1}\left(X_{i}^{2}\right) .
$$

From the definition of $\Phi_{1}$ we obtain the equalities

$$
e_{j}=\Phi^{-1}\left(X_{j}^{2}\right) \quad \text { and } \quad e_{i}=\Phi^{-1}\left(X_{i}^{2}\right)
$$

and, using condition $\left(i i_{4}\right)$ of Theorem 3.2, prove that

$$
z=\Phi_{S}^{-1}\left(X_{j} \times X_{i}\right)
$$

holds if and only if we have $z \in \mathcal{C} \backslash\{\theta\}$ and

$$
z=e_{j} * z * e_{i} .
$$

Consequently, (4.59) holds if and only if

$$
x * y=e_{j} *(x * y) * e_{i}
$$

and $x * y \in \mathcal{C} \backslash\{\theta\}$. Suppose

$$
x * y=\theta
$$


holds. From $k=j$, and (4.57), and (4.52) it follows that $x * y^{2}=x$. Now using (4.76) and Lemma 4.1, we obtain

$$
x=(x * y) * y=\theta * y=\theta .
$$

Hence, $x=\theta$ that contradicts (4.39). Consequently, we have $x * y \neq \theta$. By condition $\left(i i_{5}\right)$, from $x * y \neq \theta$ and $x \in E \cap \mathcal{C}$ and $y \in \mathcal{H} \backslash(E \cup \mathcal{C})$ it follows that $x * y \notin E$. Moreover, $x * y \in \mathcal{C}$ holds because $x \in \mathcal{C}$ and $\mathcal{C}$ is a core of $\mathcal{H}$. Consequently, the membership relation

$$
x * y \in \mathcal{C} \backslash E
$$

holds. By condition $\left(i i_{1}\right), \mathcal{C}$ belongs to $\mathbf{H}_{1}$. Now, using conditions $\left(i i_{2}\right)$ and $\left(i i_{4}\right)$ of Theorem 3.2, we obtain that there is a unique pair $i_{l}, i_{r}$ of distinct nontrivial idempotent elements of $\mathcal{C}$ such that

$$
x * y=i_{l} *(x * y) * i_{r} .
$$

Since $x$ is also a nontrivial idempotent element of $\mathcal{C}$, condition $\left(i i_{2}\right)$ of Theorem 3.2 implies $i_{l}=x=\Phi^{-1}\left(X_{j}^{2}\right)=e_{j}$. Suppose $i_{r} \neq e_{i}$. Then, using the definitions of $\Phi_{S}$ and $\Phi_{1}$, we obtain $y^{2} * i_{r}=\theta$. The last equality and (4.78) imply

$$
x * y=i_{l} *(x * y) * i_{r}=i_{l} *\left(x * y * y^{2}\right) * i_{r}=i_{l} *(x * y) * \theta=\theta,
$$

that $x * y \neq \theta$. Consequently, $i_{r}=e_{i}$ holds. Equality (4.75) follows from (4.78).

The case when condition (4.69) holds can be analyzed similarly.

The proof of the theorem is completed.

\begin{tabular}{|c|c|c|c|c|c|c|c|}
\hline & $\varnothing$ & $x y$ & $y x$ & $x^{2}$ & $y^{2}$ & $x y+y x$ & $x^{2}+y^{2}$ \\
\hline$\varnothing$ & $\varnothing$ & $\varnothing$ & $\varnothing$ & $\varnothing$ & $\varnothing$ & $\varnothing$ & $\varnothing$ \\
\hline$x y$ & $\varnothing$ & $\varnothing$ & $x^{2}$ & $\varnothing$ & $x y$ & $x y$ & $x y$ \\
\hline$y x$ & $\varnothing$ & $y^{2}$ & $\varnothing$ & $y x$ & $\varnothing$ & $y x$ & $y x$ \\
\hline$x^{2}$ & $\varnothing$ & $x y$ & $\varnothing$ & $x^{2}$ & $\varnothing$ & $x^{2}$ & $x^{2}$ \\
\hline$y^{2}$ & $\varnothing$ & $\varnothing$ & $y x$ & $\varnothing$ & $y^{2}$ & $y^{2}$ & $y^{2}$ \\
\hline$x y+y x$ & $\varnothing$ & $x y$ & $y x$ & $x^{2}$ & $y^{2}$ & $x^{2}+y^{2}$ & $x y+y x$ \\
\hline$x^{2}+y^{2}$ & $\varnothing$ & $x y$ & $y x$ & $x^{2}$ & $y^{2}$ & $x y+y x$ & $x^{2}+y^{2}$ \\
\hline
\end{tabular}

Figure 4. The Cayley table of the disjoint union of the semigroups $\mathcal{C}=\left\{\varnothing, x y, y x, x^{2}, y^{2}\right\}$ and $G=\left\{x y+y x, x^{2}+y^{2}\right\}$ with $x y=X \times Y, y x=Y \times X, x^{2}=X^{2}, y^{2}=Y^{2}, x y+y x=$ $(X \times Y) \cup(Y \times X), x^{2}+y^{2}=X^{2} \cup Y^{2}$ and $X \cap Y=\varnothing$. Here $\mathcal{C}$ is isomorphic to $\mathcal{S}_{P \otimes P}$ with $|P|=2$, and $G$ is a group of order 2, and every element of $G$ is a two-sided identity for elements of $\mathcal{C}$. 
Remark 4.7. Considering the semigroup $(\mathcal{H}, *)$ from Example 4.4 such that $\mathcal{C} \in$ $\mathbf{H}_{1}$ and every $\mathcal{H}_{\alpha}, \alpha \in \Omega$, is a group of order 2 , we see that $\mathcal{H}$ is a band of semigroups with core $\mathcal{C}$ and condition $\left(i i_{5}\right)$ of Theorem 4.6 is trivially holds. Moreover, since $E(\mathcal{C})$ is a commutative band, the definition of $(\mathcal{H}, *)$ (see $(4.3))$ implies that $E(\mathcal{H})$ is also a commutative band. Consequently. even if we have

$$
\mathcal{H} \approx\left\{\mathcal{H}_{\alpha}: \alpha \in \Omega\right\} \sqcup\{\mathcal{C}\}
$$

conditions $\left(i i_{1}\right),\left(i i_{2}\right),\left(i i_{3}\right)$, and $\left(i i_{5}\right)$ do not imply condition $\left(i i_{4}\right)$. Analogously, using Example 4.5 we can define $(\mathcal{H}, *)$ such that $(4.79)$ holds, conditions $\left(i i_{1}\right)-\left(i i_{4}\right)$ are satisfied but $\left(i i_{5}\right)$ is false (see Figure 4 for the Cayley table of corresponding $(\mathcal{H}, *))$.

Let us denote by $\mathbf{H}_{S}$ the class of all semigroups $(\mathcal{H}, *)$ satisfying condition $(i i)$ of Theorem 4.6.

Corollary 4.8. Every semigroup $(\mathcal{H}, *) \in \mathbf{H}_{S}$ admits a d-transitive monomorphism $\mathcal{H} \rightarrow \mathcal{B}_{X}$ for a suitable set $X$.

Recall that, for every semigroup $(\mathcal{S}, \circ)$, we denote by $\left(\mathcal{S}^{1}, \circ\right)$ a semigroup obtained from $(\mathcal{S}, \circ)$ by adjunction of an identity element (see $(3.23)$ ).

Theorem 4.9. Let $(L, \cdot)$ be a nonempty semigroup. The following statements are equivalent.

(i) There are a set $X$ and a partition $P$ of $X$ such that the semigroups $\left(\mathcal{S}_{P \otimes P_{S}^{1}}, \circ\right)$ and $(L, \cdot)$ are isomorphic and $|P| \geqslant 2$.

(ii) There is a semigroup $(\mathcal{H}, *) \in \mathbf{H}_{S}$ such that $(L, \cdot)$ and $\left(\mathcal{H}^{1}, *\right)$ are isomorphic.

The proof of this theorem is similar to the proof of Theorem 3.15 and we omit it here.

The following corollary can be proved similarly to Corollary 3.5 .

Corollary 4.10. Let $(\mathcal{H}, *)$ belong to $\mathbf{H}_{S}$. Then $\left(\mathcal{H}^{1}, *\right)$ admits a d-transitive monomorphism $\mathcal{H}^{1} \rightarrow \mathcal{B}_{X}$ for a suitable set $X$.

Remark 4.11. A semigroup $(\mathcal{H}, *) \in \mathbf{H}_{S}$ has an identity element if and only if $|\mathcal{H}|=7$. (The last equality holds if and only if $(\mathcal{H}, *)$ is isomorphic to $\left(\mathcal{S}_{P \otimes P_{S}}, \circ\right)$ with $|P|=2$.)

Example 4.12. Let $P=\left\{X_{0}, X_{1}, X_{2}\right\}$ be the trichotomy of the set $X=[0,1]$ defined in Example 2.8. Then the set

$$
E=\{\varnothing\} \cup\left\{R_{P}\right\} \cup\left\{X_{0}^{2}, X_{1}^{2}, X_{2}^{2}\right\} \cup\left\{X_{0}^{2} \cup X_{1}^{2}, X_{0}^{2} \cup X_{2}^{2}, X_{1}^{2} \cup X_{2}^{2}\right\}
$$


is the band of all idempotents of $\left(\mathcal{S}_{P \otimes P_{S}^{1}}, \circ\right)$. This band is commutative and it is a lattice with respect to the partial order $\leqslant$ defined by (4.2). A colored Hasse diagram of $(E, \leqslant)$ is plotted in Figure 5.

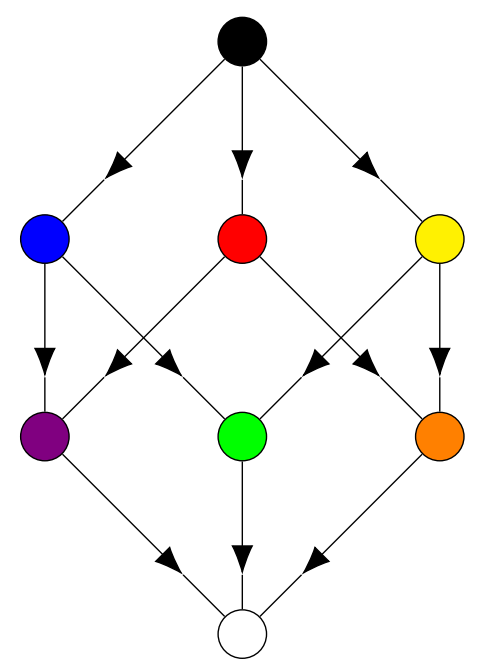

Figure 5. $R_{P}$ is white, $X_{0} \times X_{0}$ is red, $X_{1} \times X_{1}$ is yellow, $X_{2} \times X_{2}$ is blue, $X_{0}^{2} \cup X_{1}^{2}$ is orange, $X_{0}^{2} \cup X_{2}^{2}$ is green, $X_{1}^{2} \cup X_{2}^{2}$ is violet, and $\varnothing$ is black.

Let $X$ be a set, let $R$ be a nonempty binary relation on $X$. Write $X_{1}$ and $X_{2}$ for the domain and, respectively, for the range of the relation $R$, i.e., a point $x$ belongs to $X_{1}\left(X_{2}\right)$ if and only if there is $x_{2} \in X\left(x_{1} \in X\right)$ such that $\left\langle x, x_{2}\right\rangle \in R$ $\left(\left\langle x_{1}, x\right\rangle \in R\right)$.

Lemma 4.13. Let $R$ be a binary relation with a domain $X_{1}$ and a range $X_{2}$. The equality $R \circ R=\varnothing$ holds if and only if $X_{1} \cap X_{2}=\varnothing$.

Proof. It follows directly from the definition of the composition $\circ$ of binary relations.

Proposition 4.14. Let $Y$ be a set with $|Y| \geqslant 3$ and let $Q=\left\{\Delta_{Y}, \nabla_{Y}\right\}$, where $\Delta_{Y}$ is the diagonal of $Y$ and $\nabla_{Y}=Y^{2} \backslash \Delta_{Y}$. Then $Q$ is a partition of $Y^{2}$ and the subsemigroup $\left(\mathcal{S}_{Q}, \circ\right)$ of $\mathcal{B}_{Y}$ has no d-transitive representations $\mathcal{S}_{Q} \rightarrow \mathcal{B}_{X}$ for any set $X$.

Proof. It is clear that $Q$ is a partition of $Y^{2}$. Suppose there is a $d$-transitive monomorphism $\Phi: \mathcal{S}_{Q} \rightarrow \mathcal{B}_{X}$ for a suitable set $X$. Direct calculations show that

$$
\mathcal{S}_{Q}=\left\{\Delta_{Y}, \nabla_{Y}, Y^{2}\right\} \quad \text { and } \quad \nabla_{Y} \circ \nabla_{Y}=Y^{2}
$$


hold, and $\Delta_{Y}$ is the identity of $\mathcal{S}_{Q}$, and $Y^{2}$ is the zero of $\mathcal{S}_{Q}$. Let $A$ be a set of generators of $\mathcal{S}_{Q}$ for which $\Phi(A), \Phi(A)=\{\Phi(a): a \in A\}$, is a partition of $X^{2}$. Since $Y^{2}$ is a zero of $\mathcal{S}_{Q}$ and $\Phi$ is $d$-transitive, the equality $\Phi\left(Y^{2}\right)=\varnothing$ holds. It implies $Y^{2} \notin A$. There are exactly two sets, $\left\{\Delta_{Y}, \nabla_{Y}\right\}$ and $\left\{\Delta_{Y}, \nabla_{Y}, Y^{2}\right\}$, of generators of $\left(\mathcal{S}_{Q}, \circ\right)$. Consequently, we have $A=\left\{\Delta_{Y}, \nabla_{Y}\right\}$.

Let $X_{1}$ and $X_{2}$ be the domain and, respectively, the range of the relation $\Phi\left(\nabla_{Y}\right)$. We claim that the equality

$$
\Phi\left(\Delta_{Y}\right)=X_{1}^{2} \cup X_{2}^{2}
$$

holds.

Let us prove the last equality. Lemma 4.13 implies

$$
X_{1} \cap X_{2}=\varnothing
$$

and, moreover, from the definition of $X_{1}$ and $X_{2}$ it follows that

$$
\Phi\left(\nabla_{Y}\right) \subseteq X_{1} \times X_{2} .
$$

Since $\Phi$ is a monomorphism, we have

$$
\Phi\left(\nabla_{Y}\right)=\Phi\left(\Delta_{Y}\right) \circ \Phi\left(\nabla_{Y}\right)=\Phi\left(\nabla_{Y}\right) \circ \Phi\left(\Delta_{Y}\right) .
$$

Let $z$ be an arbitrary point of $X$ and let $x_{1}$ be an arbitrary point of $X_{1}$. Suppose that $z \notin X_{1} \cup X_{2}$, then (4.82) implies $\left\langle z, x_{1}\right\rangle \in \Phi\left(\Delta_{Y}\right)$ because $\left\{\Phi\left(\Delta_{Y}\right), \Phi\left(\nabla_{Y}\right)\right\}$ is a partition of $X^{2}$. Since $X_{2}$ is the range of $\Phi\left(\nabla_{Y}\right)$, there is $x_{2} \in X_{2}$ such that $\left\langle x_{1}, x_{2}\right\rangle \in \Phi\left(\nabla_{Y}\right)$. Now, using (4.83), we obtain $\left\langle z, x_{2}\right\rangle \in \Phi\left(\nabla_{Y}\right)$. Consequently, $z \in X_{1}$, that contradicts $z \notin X_{1} \cup X_{2}$. Thus, the equality $X=X_{1} \cup X_{2}$ holds. The last equality and (4.81) imply the double inclusion

$$
X^{2} \supseteq \Phi\left(\Delta_{Y}\right) \supseteq X_{1}^{2} \cup X_{2}^{2} .
$$

If the set $\Phi\left(\Delta_{Y}\right) \backslash\left(X_{1}^{2} \cup X_{2}^{2}\right)$ is nonempty, then using (4.84) and the equality $X^{2}=\Phi\left(\Delta_{Y}\right) \cup \Phi\left(\nabla_{Y}\right)$ we can find $t_{1} \in X_{1}$ and $t_{2} \in X_{2}$ such that $\left\langle t_{1}, t_{2}\right\rangle \in \Phi\left(\Delta_{Y}\right)$ or $\left\langle t_{2}, t_{1}\right\rangle \in \Phi\left(\Delta_{Y}\right)$. Without loss of generality we may suppose

$$
\left\langle t_{2}, t_{1}\right\rangle \in \Phi\left(\Delta_{Y}\right)
$$

Since $X_{1}$ is the domain of $\Phi\left(\nabla_{Y}\right)$, from $t_{2} \in X_{2}$ it follows that there is $x \in X_{1}$ such that

$$
\left\langle x, t_{2}\right\rangle \in \Phi\left(\nabla_{Y}\right) .
$$

Now (4.83), (4.85) and (4.86) give us $\left\langle x, t_{1}\right\rangle \in \Phi\left(\nabla_{Y}\right)$. Consequently, $t_{1} \in X_{2}$ holds, contrary to $t_{1} \in X_{1}$. Equality (4.80) follows.

Equality (4.80), $X=X_{1} \cup X_{2}$, and inclusion (4.82) imply

$$
X^{2} \backslash\left(\Phi\left(\Delta_{Y}\right) \cup \Phi\left(\nabla_{Y}\right)\right) \supseteq X^{2} \backslash\left(X_{1}^{2} \cup X_{2}^{2} \cup\left(X_{1} \times X_{2}\right)\right)=X_{2} \times X_{1} \neq \varnothing,
$$

i.e., $\left\{\Phi\left(\Delta_{Y}\right), \Phi\left(\nabla_{Y}\right)\right\}$ is not a partition of $X^{2}$.

Thus, contrary to our supposition, $\Phi$ is not a $d$-transitive monomorphism. 
Remark 4.15. If $|Y|=2$ and $Q=\left\{\nabla_{Y}, \Delta_{Y}\right\}$, then the equalities

$$
\Delta_{Y} \circ \nabla_{Y}=\nabla_{Y}=\nabla_{Y} \circ \Delta_{Y} \quad \text { and } \quad \nabla_{Y} \circ \nabla_{Y}=\Delta_{Y}=\Delta_{Y} \circ \Delta_{Y}
$$

hold. Consequently, $\left(\mathcal{S}_{Q}, \circ\right)$ is a group of order 2 for which the identity mapping Id: $\mathcal{S}_{Q} \rightarrow \mathcal{B}_{Y}$ is a $d$-transitive monomorphism.

The last remark shows, in particular, that the constant 3 cannot be replaced by any smaller integer in Proposition 4.14.

Acknowledgements. This research was partially supported by State Fund for Fundamental Research of Ukraine, Project F75/28173. The author thanks Prof. Ruslan Shanin, Odessa I. I. Mechnikov National University, for the useful discussions of the presented results.

\section{References}

[1] B. Albayrak, O. Givradze and G. Partenadze, Generating sets of the complete semigroups of binary relations defined by semilattices of the class $\Sigma_{2}(X, 4)$, Applied Mathematics, 9 (2018), 17-27.

[2] G. E. Andrews, The Theory of Partitions, Encyclopedia of Mathematics and its Applications, Vol. 2, Addison-Wesley Publishing Co., Reading, Mass.-LondonAmsterdam, 1976.

[3] Z. Avaliani and Sh. Makharadze, Maximal subgroups of some classes of semigroups of binary relations, Georgian Math. J., 11(2) (2004), 203-208.

[4] R. Chaudhuri and A. Mukherjea, Idempotent Boolean matrices, Semigroup Forum, 21 (1980), 273-282.

[5] A. H. Clifford, A proof of the Montague-Plemmons-Schein theorem on maximal subgroups of the semigroup of binary relations, Semigroup Forum, 1 (1970), $272-275$.

[6] A. H. Clifford and G. B. Preston, The Algebraic Theory of Semigroups. Part 1, Mathematical Surveys, No. 7, Amer. Math. Soc., Providence, R.I., 1961.

[7] H. M. Devadze, Generating sets of the semigroup of all binary relations in a finite set, Dokl. Akad. Nauk BSSR, 12 (1968), 765-768.

[8] Ya. I. Diasamidze, One-sided zeros of subsets of a semigroup of binary relations, Ukrainian Math. J., 42(5) (1990), 532-535.

[9] Ya. I. Diasamidze, One-sided units of a subset of a semigroup of binary relations, Ukrainian Math. J., 42(8) (1990), 915-918.

[10] Ya. I. Diasamidze, The structure of idempotent binary relations, Studies of semigroups (Russian), Leningrad. Gos. Ped. Inst., Leningrad, (1990), 25-28.

[11] Ya. I. Diasamidze, Right units of complete semigroups of binary relations defined by complete $X$-semilattices generated by pairwise nonintersecting sets, Bull. Georgian Acad. Sci., 166(1) (2002), 23-26. 
[12] Ya. I. Diasamidze, Right units of complete semigroups of binary relations, defined by complete X-semilattices generated by chains, Bull. Georgian Acad. Sci., 167(2) (2003), 197-199.

[13] Ya. Diasamidze and Sh. Makharadze, Complete Semigroups of Binary Relations, Kriter, Turkey, 2013.

[14] Ya. I. Diasamidze, N. Aydin and A. Erdoğan, Generating set of the complete semigroups of binary relations, Applied Mathematics, 7 (2016), 98-107.

[15] O. Givradze, Some properties of semigroup $B_{X}(D)$, defined by semilattice of $\Sigma_{1}(X, 4)$ class, Bull. Georgian Acad. Sci., 167(1) (2003), 43-46.

[16] O. Givradze, Y. Diasamidze and N. Tsinaridze, Generated sets of the complete semigroup binary relations defined by semilattices of the finite chains, Trans. A. Razmadze Math. Inst., 172 (2018), 378-387.

[17] P. Hell and J. Nešetřil, Graphs and Homomorphisms, Oxford Lecture Series in Mathematics and its Applications, Vol. 28, Oxford University Press, Oxford, 2004.

[18] P. M. Higgins, Techniques of Semigroup Theory, with a foreword by G. B. Preston, Oxford Science Publications, The Clarendon Press, Oxford University Press, New York, 1992.

[19] J. M. Howie, Fundamentals of Semigroup Theory, London Mathematical Society Monographs, New Series, 12, Oxford Science Publications, The Clarendon Press, Oxford University Press, New York, 1995.

[20] W. Imrich, S. Klavžar and D. F. Rall, Topics in Graph Theory: Graphs and Their Cartesian Product, A K Peters, Ltd., Wellesley, MA, 2008.

[21] K. H. Kim and F. W. Roush, Inverses of Boolean matrices, Linear Algebra Appl., 22 (1978), 247-262.

[22] F. Klein-Barmen, Über eine weitere verallgemeinerung des verbandsbegriffes, Math. Z., 46 (1940), 472-480.

[23] J. Konieczny, Reduced idempotents in the semigroup of Boolean matrices, J. Symbolic Comput., 20 (1995), 471-482.

[24] J. Konieczny, A proof of Devadze's theorem on generators of the semigroup of Boolean matrices, Semigroup Forum, 83(2) (2011), 281-288.

[25] K. Kuratowski and A. Mostowski, Set Theory, with an Introduction to Descriptive Set Theory, Translated from the 1966 Polish original, Second, completely revised edition, Studies in Logic and the Foundations of Mathematics, Vol. 86, North-Holland Publishing Co., Amsterdam-New York-Oxford; PWNPolish Scientific Publishers, Warsaw, 1976.

[26] D. B. McAlister, Homomorphisms of semigroups of binary relations, Semigroup Forum, 3(2) (1971/72), 185-188. 
[27] R. McKenzie and B. M. Schein, Every semigroup is isomorphic to a transitive semigroup of binary relations, Trans. Amer. Math. Soc., 349(1) (1997), 271285.

[28] J. S. Montague and R. J. Plemmons, Maximal subgroups of the semigroup of relations, J. Algebra, 13 (1969), 575-587.

[29] C. Namnak and P. Preechasilp, Natural partial orders on the semigroup of binary relations, Thai J. Math., 4(3) (2006), 39-50.

[30] O. Ore, Theory of equivalence relations, Duke Math. J., 9 (1942), 573-627.

[31] R. J. Plemmons and B. M. Schein, Groups of binary relations, Semigroup Forum, 1 (1970), 267-271.

[32] R. J. Plemmons and M. T. West, On the semigroup of binary relations, Pacific J. Math. 35 (1970), 743-753.

[33] G. B. Preston, Any group is a maximal subgroup of the semigroup of binary relations on some set, Glasgow Math. J., 14 (1973), 21-24.

[34] B. M. Schein, Regular elements of the semigroup of all binary relations, Semigroup Forum, 13(2) (1976/77), 95-102.

[35] B. M. Shain, Representation of semigroups by means of binary relations, Mat. Sb. (N.S.), 60(102) (1963), 293-303.

[36] T. Tamura, Operations on binary relations and their applications, Bull. Amer. Math. Soc., 70(1) (1964), 113-120.

[37] P. M. Whitman, Lattices, equivalence relations and subgroups, Bull. Amer. Math. Soc., 52 (1946), 507-522.

[38] K. A. Zaretskii, Regular elements of the semigroup of binary relations, Uspehi Mat. Nauk, 17 (1962), 177-179.

[39] K. A. Zaretskii, The semigroup of binary relations, Mat. Sb. (N.S.), 61(103) (1963), 291-305.

\section{O. Dovgoshey}

Function Theory Department

Institute of Applied Mathematics and Mechanics of NASU

Dobrovolskogo Str. 1, Slovyansk 84100, Ukraine

e-mail: oleksiy.dovgoshey@gmail.com 